\title{
Fault Detection of High-Speed Train Wheelset Bearing Based on Impulse-Envelope Manifold
}

\author{
Zhe Zhuang, ${ }^{1}$ Jianming Ding, ${ }^{1}$ Andy C. Tan, ${ }^{2}$ Ying Shi, ${ }^{1}$ and Jianhui Lin ${ }^{1}$ \\ ${ }^{1}$ State Key Laboratory of Traction Power, Southwest Jiaotong University, Chengdu 610031, China \\ ${ }^{2}$ LKC Faculty of Engineering and Science, Universiti Tunku Abdul Rahman, 43000 Kajang, Malaysia \\ Correspondence should be addressed to Jianming Ding; fdingjianming@126.com
}

Received 26 May 2017; Accepted 12 October 2017; Published 3 December 2017

Academic Editor: Sara Muggiasca

Copyright (c) 2017 Zhe Zhuang et al. This is an open access article distributed under the Creative Commons Attribution License, which permits unrestricted use, distribution, and reproduction in any medium, provided the original work is properly cited.

\begin{abstract}
A novel fault detection method employing the impulse-envelope manifold is proposed in this paper which is based on the combination of convolution sparse representation (CSR) and Hilbert transform manifold learning. The impulses with different sparse characteristics are extracted by the CSR with different penalty parameters. The impulse-envelope space is constructed through Hilbert transform on the extracted impulses. The manifold based on impulse-envelope space (impulse-envelope manifold) is executed to learn the low-dimensionality intrinsic envelope of vibration signals for fault detection. The analyzed results based on simulations, experimental tests, and practical applications show that (1) the impulse-envelope manifold with both isometric mapping (Isomap) and locally linear coordination (LLC) can be successfully used to extract the intrinsic envelope of the impulses where local tangent space analysis (LTSA) fails to perform and (2) the impulse-envelope manifold with Isomap outperforms those with LLC in terms of strengthening envelopes and the number of extracted harmonics. The proposed impulse-envelope manifold with Isomap is superior in extracting the intrinsic envelope, strengthening the amplitude of intrinsic envelope spectra, and enlarging the harmonic number of fault-characteristic frequency. The proposed technique is highly suitable for extracting intrinsic envelopes for bearing fault detection.
\end{abstract}

\section{Introduction}

Train bogie is one of the crucial subsystems of high-speed train and consists of many critical mechanical components, one of which is wheelset bearing. Due to the demanding working conditions surrounding the wheelset bearing (such as alternating contact stresses, heavy loads, and wheel-rail shock), the faults on the component surface of wheelset bearing are easily generated. The vibration and shock excited by the bearing faults could endanger the running safety of the high-speed train. Thus, it is important to detect symptom of its faults at an early stage to ensure safe operation of the train.

The traditional detection of bearing faults mainly involves grease or oil monitoring, acoustic emission techniques, hotbox detection, and vibration signal monitoring [1]. The standards require a level-three maintenance approval of highspeed train certification before the wheelset bearing can be disassembled. This makes grease or oil monitoring unsuitable for wheelset bearing fault detection. The attenuation of transient elastic waves generated by a bearing fault restricts wide application of acoustic emission techniques in bearing fault detection [2]. Hotbox detection is commonly used in practice. When the temperature of bearing housing exceeds a preset threshold the hotbox detector sets an alarm. However, hotbox detector is only sensitive to severe faults, for example, bearing burn-off [1]. Not only can the axle-box acceleration signals be conveniently and reliably measured at relatively low cost, but also they carry vital information about the health state of the bearing faults $[3,4]$. Consequently, vibration signal monitoring has become an effective and feasible detection technique for wheelset bearing faults, especially at the incipient stage.

The impulse series or periodic impulses embedded in the bearing vibration signals carry essential information of the bearing condition. The amplitudes of these impulses induced by bearing faults are modulated by the variances of the position and direction of forces applied on rollers $[5,6]$. The fault-characteristic frequencies of the bearing are modulated 
by the slippage of rolling elements and the fluctuation of wheelset rotational speeds $[7,8]$. The impulses generated by bearing faults are transient impulses containing a wide range of frequencies with very short time duration. This can easily excite multimodel resonance responses of the structure and sensor [9]. The impulses generated by bearing faults are usually masked by background noises and structural vibration [10, 11]. In addition, the impulses generated by bearing faults are mixed with those caused by the wheelrail interaction when the wheel tread has defects or is out of roundness. These nonlinear and nonstationary modulation characteristics, multimodel effects, noise, interferences, and impulse-mixture make it difficult and complex to extract these impulses from the measured vibration signals. Extracting impulses has long been a challenge in the field of fault detection and diagnosis.

To effectively tackle this problem, many advanced signal processing techniques have been proposed, including empirical model decomposition (EMD) $[12,13]$, wavelet transform (WT) $[14,15]$, and compressive sensing (CS) [16, 17]. Among them, EMD is suitable for analyzing nonlinear and nonstationary signals and models the analyzed signal as the sum over a set of intrinsic model functions (IMFs) and a residual [12]. EMD has been successfully applied in the field of fault diagnosis, failure detection, damage identification, and health monitoring $[18,19]$. The shortcomings of EMD are the lack of theoretical foundation [20], sensitivity to noises, sampling errors [21], and model mixtures [10]. These have adverse effects on the performance for fault detection. To alleviate these problems, the variants of EMD, such as EEMD, bivariate empirical mode decomposition (BEMD), and multivariate empirical mode decomposition (MEMD) [22-24], have been developed. Moreover, there is a lack of theory to clarify these problems. Wavelet analysis is conducted by the inner product of the measured vibration signals and wavelet basis provides the time-scale information of the impulses. Both continuous wavelet transform (CWT) and discrete wavelet transform (DWT) have been successfully applied for fault detection of rotational machines with multiresolution $[25,26]$. The huge computational costs and redundant coefficients of CWT limit its wide application as compared to DWT which has a faster algorithm [27]. Wavelet packet transform (WPT) is proposed to improve the decomposition performance of WT on highfrequency band which contains useful information for fault detection [28]. However, the decomposition quality of the DWT and WPT is influenced by the selection of mother wavelet [29]. The shift-variance [30] and the low oscillation [31] of DWT and WPT result in distortion and contain nonsparse representation of the impulses.

CS consists of sparse representation and dictionary construction. With sparse representation models, the signals are the linear combinations of basis elements or atoms in a redundant dictionary. Dictionary design is adapted to selffeature the vibration signals to match well with the high-level structures of the impulses. As far as sparse representation is concerned, the exact resolution of sparse representation proves to be an NP-hard problem [32], and the approximate solutions based on greedy-based matching pursuit [33] and convex optimal-based basis pursuit [34] are considered instead. Dictionary construction includes manually predefined dictionary and dictionary learning. Dictionary learning includes regular dictionary learning [35] and shiftinvariant dictionary learning (SIDL) $[36,37]$. For excellent performances of CS on representing signals (such as selfadaption, sparsity, and super-resolution), the CS based on the predefined dictionaries and sparse representation is used to detect faults on bearings and gear boxes and outperforms EMD and WT $[38,39]$. Compared to predefined dictionary and regular dictionary learning, SIDL has shift-invariance $[36,37]$.

The convolution sparse representation (CSR) based on alternating direction method of multipliers (ADMM) was first proposed in 2016 and is a novel research on CS based on pursuit and SIDL [38]. CSR provides an excellent framework for extracting impulses induced by bearing faults and wheel-rail interactions. However, the performances of CSR on extracting impulses are heavily influenced by its penalty parameter which determines the sparsity of the extracted impulses. When the penalty parameter is very large, the extracted impulse is very sparse and the number of the extracted impulses is far less than the true number of impulses [39]. Thus, this can easily lead to the loss of impulses containing fruitful fault-information. Conversely, the extracted impulses could be far more than the true number of impulses. As such, this results in impulse corruptions. To completely extract impulses with the least corruptions is very difficult under the condition of a single penalty parameter. Hence, in this paper, the CSRs with different penalty parameters are jointly used to extract impulses in the framework of manifold learning.

Manifold learning projects the high-dimensional data into a lower-dimensional feature space by preserving the local neighborhood structures. Manifold learning mainly involves convex and nonconvex techniques. Convex technique involves full spectrum methods, such as isometric mapping (Isomap) [40], diffusion maps [41], and sparse spectral methods (which include local tangent space analysis (LTSA) [42] and local linear embedding (LLE) [43]). Nonconvex technique mainly includes locally linear coordination (LLC) [44] and Sammon mapping [45]. Recently, manifold learning has been successfully applied in fault diagnosis. The manifolds based on different spaces, such as scale space of WT and WPT [46, 47], IMF space of EMD [48], and timefrequency space [49], are used to extract impulses. These manifold methods have successfully discovered the intrinsic structures of impulses embedded in the measured vibration signals.

Hence, this paper aims to fully exploit the framework for extracting impulses with CSR which provides lower or minimum adverse influences that the inappropriate selection of penalty parameter of CSR brings. This novel fault detection method known as impulse-envelope manifold is proposed based on the combination of CSR, Hilbert transform, and manifold learning. The impulses with different sparsity characteristic are extracted by the CSR with different penalty parameters. The impulse-envelope spaces are spanned by the Hilbert transform on the extracted impulses. The manifold 
based of the impulse-envelope spaces is used to discover the low-dimensionality intrinsic envelope of bearing faults impulses embedded in vibration signals for fault detection. The results show that the proposed method is capable of detecting bearing faults through simulations, experimental tests, and a practical test. This paper is organized as follows. The basic theory of impulse-envelope manifold is introduced in Section 2. Simulation validation is executed in Section 3. Experiment verifications are demonstrated in Section 4. Section 5 illustrates the practical application of the proposed method. Section 6 provides the conclusion of the paper.

\section{Basic Theory of Impulse-Envelope Manifold}

2.1. Impulse Extraction by CSR. CSR models a signal as the sum of a set of convolutions of atoms with the sparse coefficients defined as

$$
\begin{aligned}
\left\{\mathbf{d}_{m}, \mathbf{x}_{k, m}\right\}= & \underset{\left\{\mathbf{d}_{m}, \mathbf{x}_{k, m}\right\}}{\arg \min } \frac{1}{2} \sum_{k=1}^{K}\left\|\mathbf{s}_{k}-\sum_{m=1}^{M} \mathbf{d}_{m} * \mathbf{x}_{k, m}\right\|_{F}^{2} \\
& +\lambda \sum_{k=1}^{K} \sum_{m=1}^{M}\left\|\mathbf{x}_{k, m}\right\|_{1},
\end{aligned}
$$

where $\mathbf{s}_{k} \in \mathbb{R}^{n}$ are $k$ th analyzed vibration signals, $n$ is length of analyzed signal, $\mathbf{d}_{m} \in \mathbb{R}^{p}$ is a set of atoms, $p$ is length of atom, $\mathbf{x}_{k, m} \in \mathbb{R}^{n-p+1}$ are sparse coefficients related to a given signal $\mathbf{s}_{k}$ and atom $\mathbf{d}_{m}, \lambda \in \mathbb{R}^{+}$is penalty parameter, $K$ is number of interval signals $\mathbf{s}_{k}$, and $M$ is number of atoms $\mathbf{d}_{m}$.

The sparse coefficients $\mathbf{x}_{k, m}$ are resolved by the shiftinvariance sparse coding (SISC) of (1), when the signals $\mathbf{s}_{k}$ and atoms $\mathbf{d}_{m}$ are given. The SISC can be expressed as

$$
\begin{aligned}
\mathbf{x}_{k, m}= & \arg \min \frac{1}{2} \sum_{k=1}^{K}\left\|\mathbf{s}_{k}-\sum_{k} \mathbf{d}_{m} * \mathbf{x}_{k, m}\right\|_{F}^{2} \\
& +\lambda \sum_{k=1}^{K} \sum_{m=1}^{M}\left\|\mathbf{x}_{k, m}\right\|_{1} .
\end{aligned}
$$

In (2), the different sparse coefficients $\mathbf{x}_{k, m}$ are decoupled with respect to different analyzed signals $\mathbf{s}_{k}$; the sparse coefficients $\mathbf{x}_{m}$ corresponding to signals $\mathbf{s}$ can be individually solved by

$$
\mathbf{x}_{m}=\underset{\mathbf{x}_{m}}{\arg \min } \frac{1}{2}\left\|\mathbf{s}-\sum_{k} \mathbf{d}_{m} * \mathbf{x}_{m}\right\|_{F}^{2}+\lambda \sum_{m=1}^{M}\left\|\mathbf{x}_{m}\right\|_{1} .
$$

The atoms $\mathbf{d}_{m}$ are obtained by SIDL using (1), when the signal $\mathbf{s}_{k}$ and sparse coefficients $\mathbf{x}_{k, m}$ are known. The SIDL is described as

$$
\mathbf{d}_{m}=\underset{\mathbf{d}_{m}}{\arg \min } \frac{1}{2} \sum_{k=1}^{K}\left\|\mathbf{s}-\sum_{k} \mathbf{d}_{m} * \mathbf{x}_{k, m}\right\|_{F}^{2},
$$

such that $\left\|\mathbf{d}_{m}\right\|=1 \forall m$.

The details on SISC and SIDL of CSR based on alternating direction method of multipliers (ADMM) can be found in [38]. The atoms $\mathbf{d}_{m}^{\lambda}$ are learned from the interval signals $\mathbf{s}_{k} \in \mathbb{R}^{n}(k=1,2, \ldots, K)$ through interleaved iterations of SISC and SIDL of CSR with different penalty parameters $\lambda$. The sparse coefficients $\mathbf{x}_{m}^{\lambda}$ associated with the signals $\mathbf{s}=\left[\mathbf{s}_{1}{ }^{T}, \ldots, \mathbf{s}_{K}{ }^{T}\right]^{T} \in \mathbb{R}^{n K}$ are obtained through SISC based on the learned atoms $\mathbf{d}_{m}^{\lambda}$. Hence, the impulses $\mathbf{I}_{m}^{\lambda}$ with different sparsity characteristic are extracted by the convolution of atoms $\mathbf{d}_{m}^{\lambda}$ and associated sparse coefficients $\mathbf{x}_{m}^{\lambda}$ and are expressed as

$$
\mathbf{I}_{m}^{\lambda}=\sum_{m=1}^{M} \mathbf{d}_{m}^{\lambda} * \mathbf{x}_{m}^{\lambda}
$$

2.2. Impulse-Envelope Space Construction. The characteristic information on bearing faults is contained in the envelope of the extracted impulses. The Hilbert transform $\mathbf{h}_{m}^{\lambda}$ of the extracted impulses $\mathbf{I}_{m}^{\lambda}$ is defined as [50]

$$
\mathbf{h}_{m}^{\lambda}=\frac{2}{\pi} \int_{-\infty}^{\infty} \frac{\mathbf{I}_{m}^{\lambda}(t)}{t-\tau} d \tau=\frac{1}{\pi t} \mathbf{I}_{m}^{\lambda}(t) .
$$

The Hilbert transform can be viewed as a filter of unity amplitude and phase $\pm 90^{\circ}$ depending on the sign of the frequency of input signal spectrum. The real signal $\mathbf{I}_{m}^{\lambda}$ and its Hilbert transform $\mathbf{h}_{m}^{\lambda}$ can form a complex signal, called the analytical signal:

$$
\mathbf{z}_{m}^{\lambda}=\mathbf{I}_{m}^{\lambda}+\mathbf{j h}_{m}^{\lambda}
$$

The envelope $\mathbf{E}_{m}^{\lambda}$ of the complex signal $\mathbf{z}_{m}^{\lambda}$ is defined as

$$
\mathbf{E}_{m}^{\lambda}=\left|\mathbf{I}_{m}^{\lambda}+\mathbf{j} \mathbf{h}_{m}^{\lambda}\right|=\sqrt{\left(\mathbf{I}_{m}^{\lambda}\right)^{2}+\left(\mathbf{h}_{m}^{\lambda}\right)^{2}} .
$$

Suppose $\lambda_{i}=\lambda_{1}+0.5(i-1)(i=1,2, \ldots, N)$ and $\lambda_{1}=$ 1 in this paper; $N$ is determined when the number of the nonzero elements of sparse coefficients $\mathbf{x}_{m}^{\lambda_{N+0.5}}$ equals zero. The impulse-envelope space IES is spanned by all the envelopes, $\mathbf{z}_{m}^{\lambda_{i}}$, and expressed as

$$
\mathrm{IES}_{m}=\left[\begin{array}{llll}
\mathbf{E}_{m}^{\lambda_{1}} & \mathbf{E}_{m}^{\lambda_{2}} & \cdots & \mathbf{E}_{m}^{\lambda_{N}}
\end{array}\right] \in \mathbb{R}^{n K \times N} .
$$

2.3. Manifold Learning Based on Impulse-Envelope Space. The impulse-envelope manifold transforms the impulse-envelope space IES $_{m}$ with high-dimensionality $N$ in (9) into a new intrinsic impulse-envelope space $\mathbf{I}_{m}$ with dimension $d$, while retaining the geometry structure of the impulse-envelope

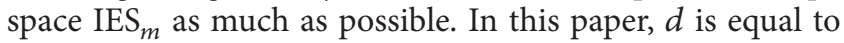
1 because there is only one-type impulse in every impulseenvelope space. Manifold learning involves convex and nonconvex techniques. Convex technique includes full spectrum and sparse spectral methods. All manifold leaning has its advantages and shortcomings. In this paper the manifold learning based on impulse-envelope space is conducted for the first time, and no references are available. Hence, the Isomap with full spectrum methods, the LTSA in sparse spectral methods, and the LLC in nonconvex techniques are 


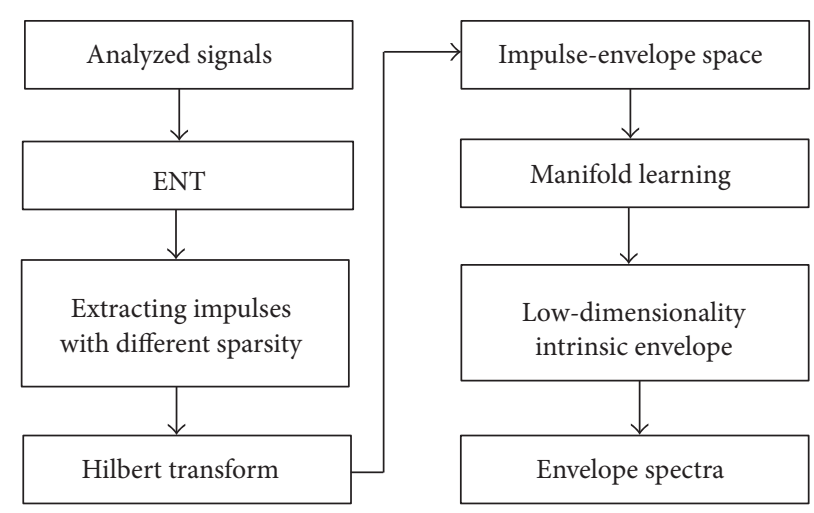

FIGURE 1: Fault detection flowchart of the impulse-envelope manifold.

selected to learn the low-dimensionality intrinsic structures of the impulse-envelope embedded in high-dimensionality impulse-envelope space. The detailed algorithms of Isomap, LTSA, and LLC can be found in [40], [42], and [44], respectively. In addition, the nearest neighbors $\kappa$ in manifold learning are set as the length of atom $p$.

2.4. Estimation of the Number of the Atom Type (ENA). The number of atoms $M$ is not determined in (1); instead it is based on prior vibration mechanism of the impulses generated by bearing faults [5]. An impulse can be completely described by two parameters, namely, the resonance frequency and damping coefficient. The resonance frequency can identify different atoms. So the dominant frequencies of the atoms are used to estimate the number of atoms $M$. Considering the defects of outer race, inner race, roller, and wheel tread, generally, the number $M$ is preset as 4 in wheelset bearing fault detection. The true value of $M$ is obtained via iteratively executing the CSR. The dominant frequencies $f_{m}(m=1,2, \ldots, M)$ of each atom are extracted by Fourier transform. If the difference of any two dominant frequencies is more than the frequency resolution $f_{s} p^{-1}\left(f_{s}\right.$ denotes the sampling frequency), this illustrates that there are $M$ atoms. Otherwise, $M=M-1$ and the operation is repeated to execute CSR and calculate the dominant frequencies until the difference of any two dominant frequencies is more than the frequency resolution. The final value of $M$ is output as the estimated value of the number of atoms.

2.5. Fault Detection Based on Impulse-Envelope Manifold. The schematic of fault detection based on impulse-envelope manifold is summarized and shown in Figure 1.

The proposed impulse-envelope manifold method includes the following.

Step 1. Input the analyzed signals.

Step 2. Estimate the number of atom types using dominant frequency analysis.

Step 3. Extract the impulses with different sparsity characteristic using (1) to (5).
Step 4. Construct the impulse-envelope space using (9).

Step 5. Execute the manifold learning based on impulseenvelope space to learn the low-dimensionality intrinsic envelope.

Step 6. Use envelope spectra of the intrinsic envelope to judge the bearing fault.

\section{Simulation Validation}

To illustrate the effectiveness of the proposed method, the acceleration responses $a(t)$ induced by a bearing fault are simulated using the impulse function of a mass-spring-damper system [5] and are expressed as

$$
a(t)=\sum_{l=1}^{L} A_{l} e^{-\beta\left(t-l T_{p}\right)} \cos \left[\omega\left(t-l T_{p}\right)\right] u\left(t-m l T_{p}\right),
$$

where $A_{l}$ represents the amplitude of the $l$ th fault impulse, which is set as $0.000000005, T_{p}$ represents the reciprocal of fault-character frequency $f_{p}$, which is set as $49.1 \mathrm{~Hz}, \beta$ is the structural damping coefficient, set as $1200 \mathrm{Ns} / \mathrm{m}, \omega$ is the nature frequency, which is $2000 \mathrm{~Hz}$, and $L$ represents the number of the simulated impulses, which is 39.

The fault simulation signal with $-7 \mathrm{~dB}$ signal-to-noise ratio (SNR) is shown in Figure 2, which are generated by mixing the fault response in (10) with white noises. The low-dimensionality intrinsic impulse-envelopes are extracted from the noise signals as shown in Figure 3(b) and by using the proposed impulse-envelope manifold shown in Figure 3(d). Through comparisons, the manifolds with Isomap and LLC can successfully extract the impulse-envelope of the noise signals based on the impulse-envelope space; the number of the extracted impulse-envelopes is 39 which are shown in Figures 3(a) and 3(c) and is equal to the preset value $L$ of simulation impulses. However, LTSA fails to learn the impulse-envelope from the impulse-envelope space. Meanwhile, the fault-characteristic frequency $f_{p}$ and its 20order harmonics are extracted by the spectra of the intrinsic impulse-envelope as shown in Figures 3(b) and 3(d). The amplitudes of the extracted intrinsic envelope by Isomap are larger than the ones by LLC; as a result, the amplitudes 


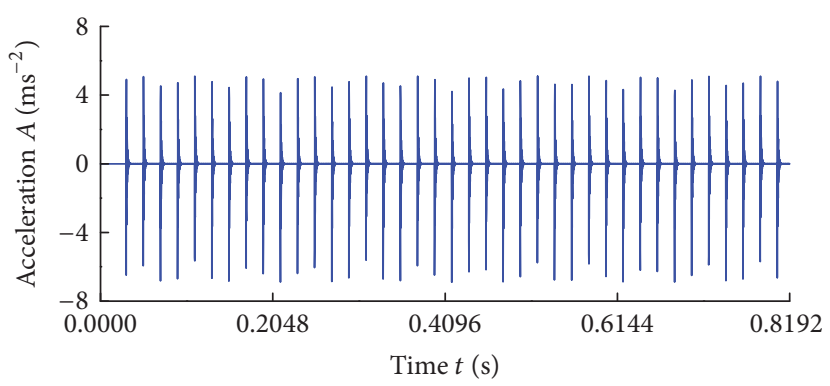

(a)

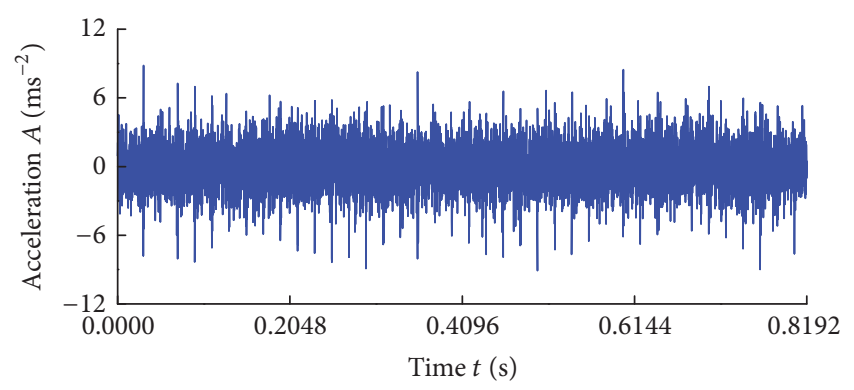

(b)

FIgURE 2: Simulation signals: (a) the original signals with periodic impulses and (b) the signals with added white noise (SNR $=-7 \mathrm{~dB})$.

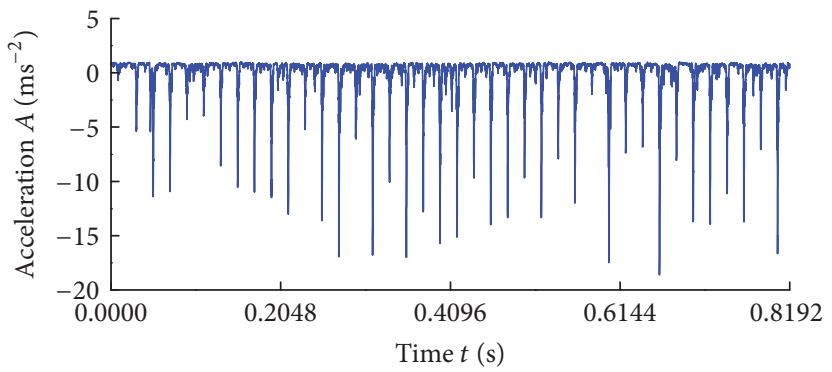

(a)

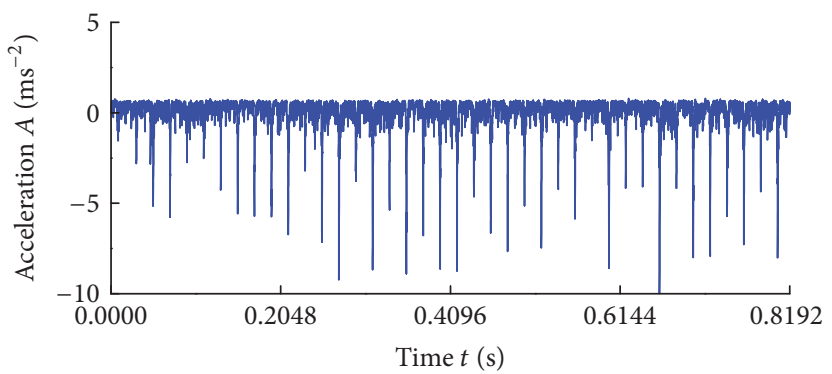

(c)

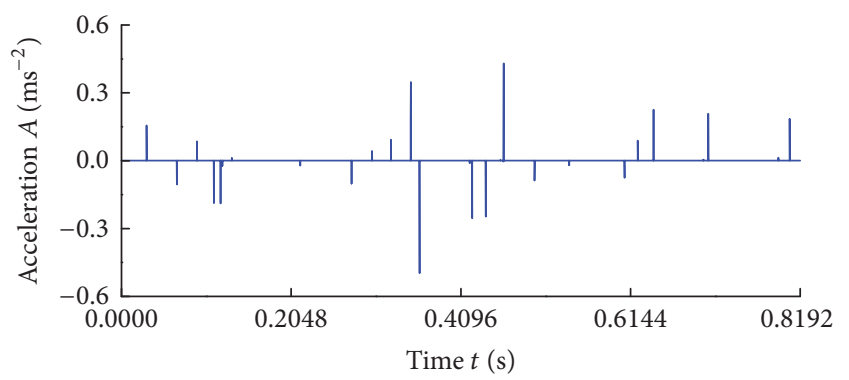

(e)

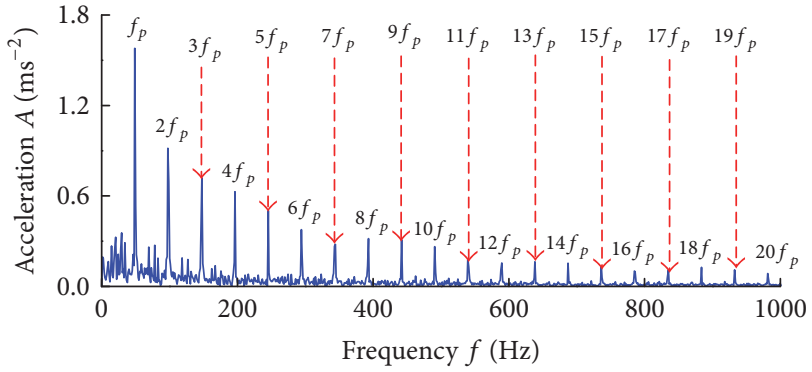

(b)

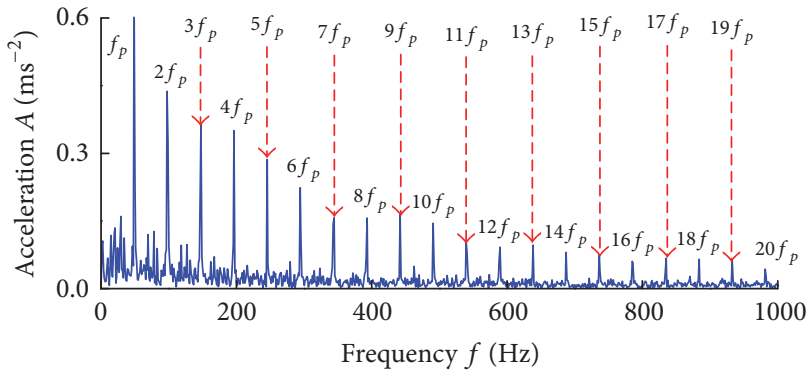

(d)

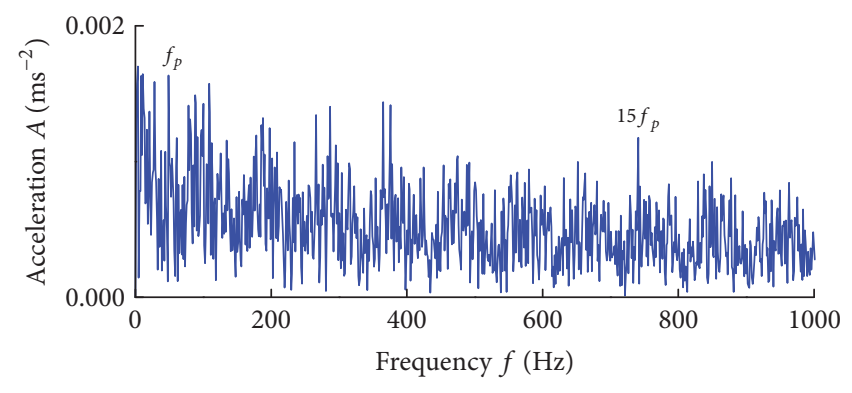

(f)

FIGURE 3: The intrinsic impulse-envelopes extracted by the impulse-envelope manifold and their envelope spectra: (a) impulse-envelope with Isomap and (b) the envelope spectra of (a), (c) impulse-envelope with LLC and (d) the envelope spectra of (c), (e) impulse-envelope with LTSA, and (f) the envelope spectra of (e).

of the envelope spectra by Isomap are also larger than the ones obtained by LLC. This shows that the impulse-envelope manifold with Isomap has a better performance in extracting the envelopes than the LLC.

To compare the manifolds with Isomap based on two other envelope spaces spanned by the Hilbert transform of
IMFs in EEMD and multiscale decomposition signals in WPT, the decomposition results obtained by EEMD and WPT from processing the signals in Figure 2(b) are shown in Figures 4 and 5, respectively. The number of ensembles and the amplitude of the added white noise are two parameters needed to be set when the EEMD method is used. Generally, 

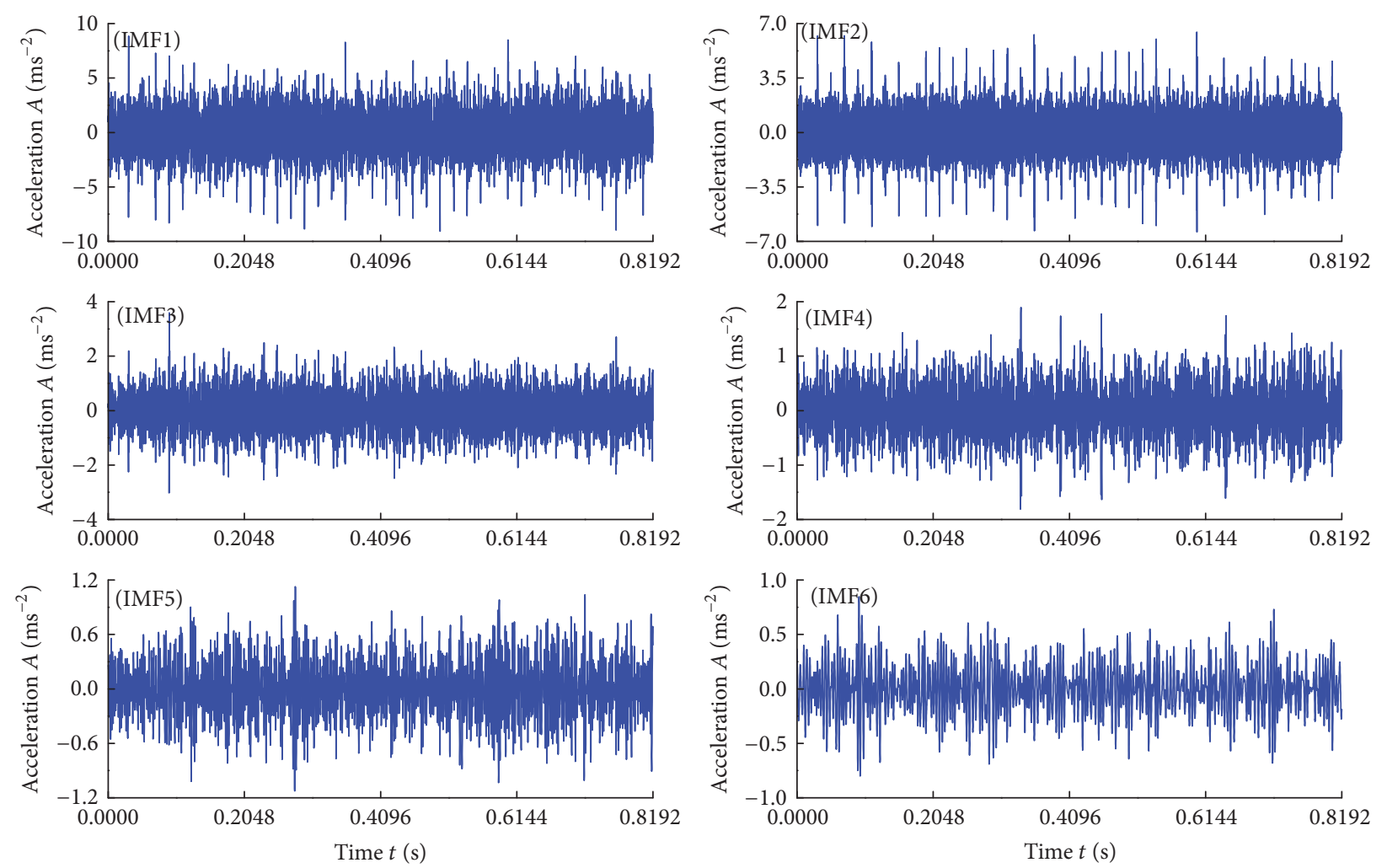

FIGURE 4: IMFs obtained by EEMD.

when the signal is dominated by high-frequency components, the noise amplitude needs to be smaller. And when the signal is dominated by low-frequency components, the noise amplitude should be increased. In this paper, the amplitude of the added white noise is 0.2 times the standard deviation of the signal, and the number of ensembles is set as 400 according to $N=(a / e)^{2}$, where $N$ is the ensemble number, $a$ is the amplitude of the added white noise, and $e$ is the standard deviation of error, which is defined as 0.01 [51]. Daubechies wavelet (Db8) is selected as the mother wavelet of WPT due to its wide application in engineering signal processing $[52,53]$. The intrinsic impulse-envelopes are extracted by the manifold with Isomap based on both IMF-envelope and multiscale envelope spaces, and the results are shown in Figure 6. The IMF-envelope space extracted 12 harmonics of the faultcharacteristic frequency while multiscale envelope space has 5 -order harmonics (the number of harmonics is lower than 20 in the impulse-envelope space). The amplitudes of the envelope and envelope spectra obtained by the manifold with Isomap based on IMF-envelope and multiscale envelope space are less than the impulse-envelope space with Isomap and are almost the same as the impulse-envelope space with LLC. These results show that the impulse-envelope space outperforms the IMF-envelope and multiscale envelope space in extracting the intrinsic impulse-envelope.

\section{Experimental Verification}

Figure 7 shows the wheelset bearing test bench used for the practical tests. The test bench consists of a motor, driving
TABLE 1: The parameters of the test rolling bearing.

\begin{tabular}{lccc}
\hline $\begin{array}{l}\text { Roller } \\
\text { number }\end{array}$ & $\begin{array}{c}\text { Roller diameter } \\
(\mathrm{mm})\end{array}$ & $\begin{array}{c}\text { Pitch diameter } \\
(\mathrm{mm})\end{array}$ & $\begin{array}{c}\text { Contact angle } \\
(\mathrm{rad})\end{array}$ \\
\hline 19 & 26.9 & 180 & 0.1571 \\
\hline
\end{tabular}

wheel, loading device, wheelset, and axle box. The motor delivers the driving power with different motor speeds. The driving power is conveyed to the driving wheel through rubber belts. The traction power of the driving wheel is then transmitted to the wheelset. The artificial faults are introduced on the bearing outer race and on a roller as shown in Figure 8. The artificially faulted roller bearing is installed in the axle box and an accelerometer is mounted on the housing. The parameters of the faulty bearing are listed in Table 1.

\subsection{Result and Discussion: Fault Detection with an Outer} Race Fault. The vibration signals measured from the wheelset bearing test bench are shown in Figure 9 and those with an artificially simulated fault on the bearing outer race are shown in Figure 8(a).

The intrinsic impulse-envelopes learned by the impulseenvelope manifold with Isomap, LLC, and LTSA are shown in Figure 10. The impulse-envelope manifold with Isomap and LLC successfully detects the fault-characteristic frequency of an outer race defect and with 7 harmonics. However, the impulse-envelope manifold with LTSA fails to extract the 

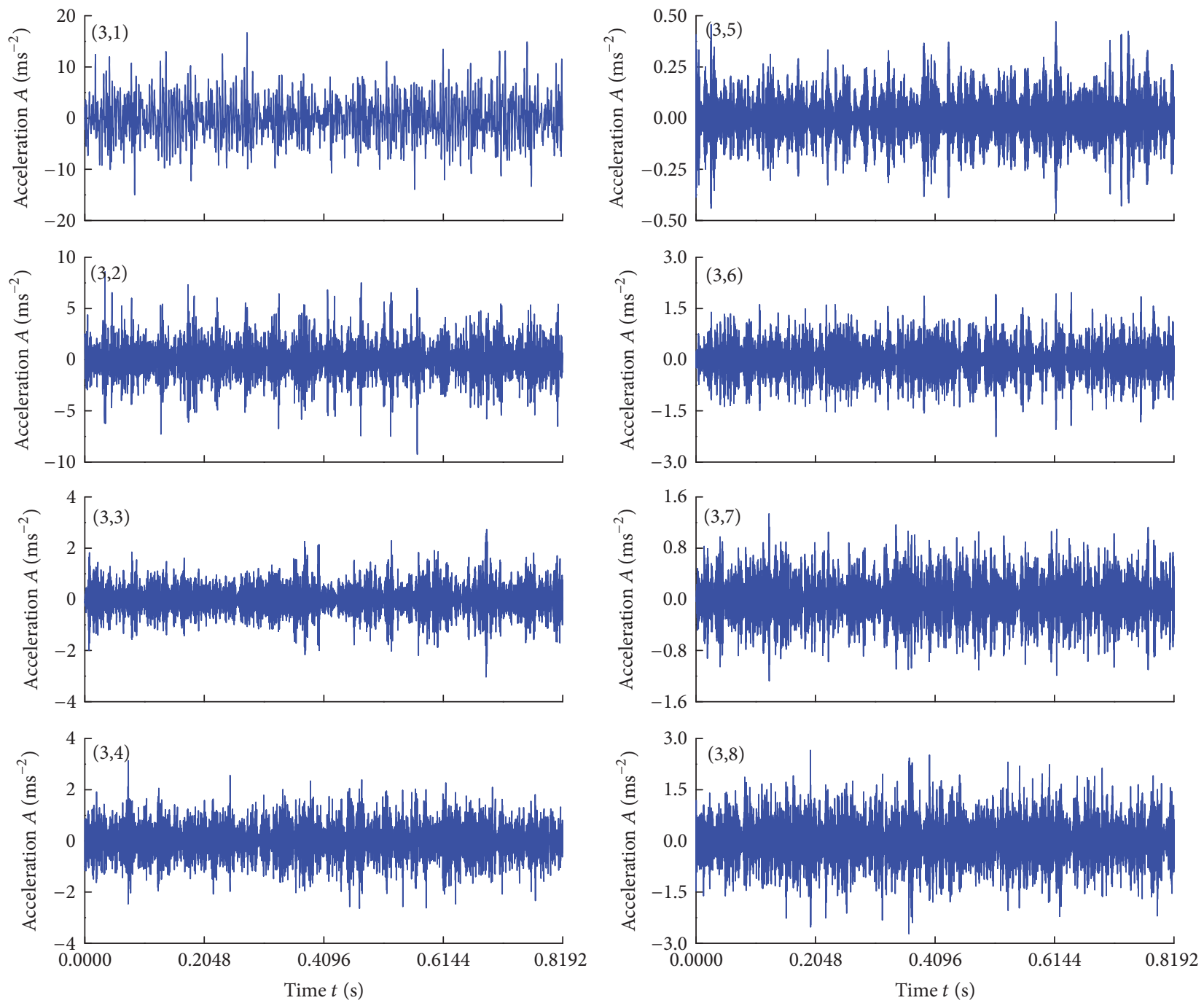

FIGURE 5: Multiscale signals obtained by WPT.

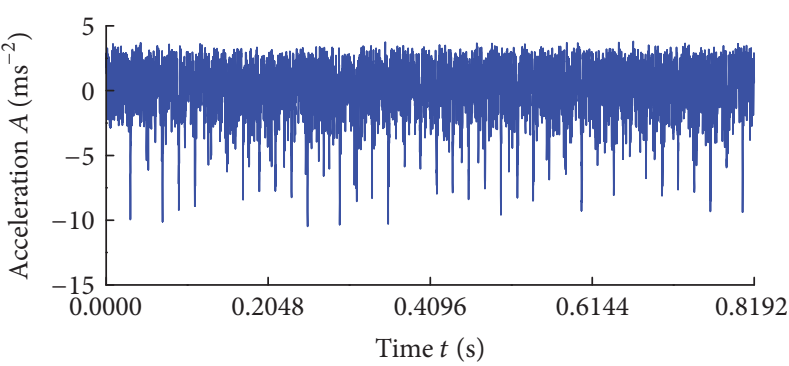

(a)

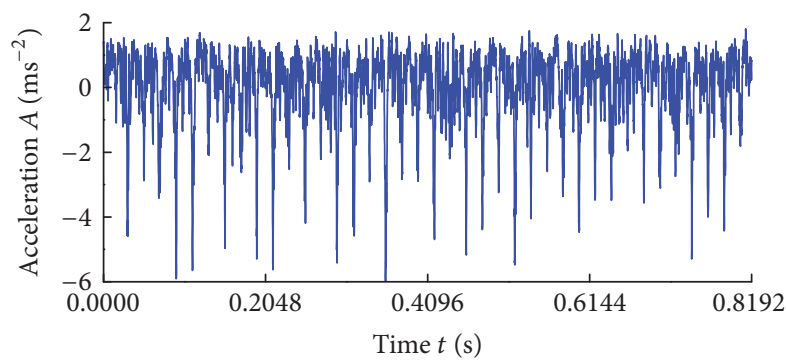

(c)

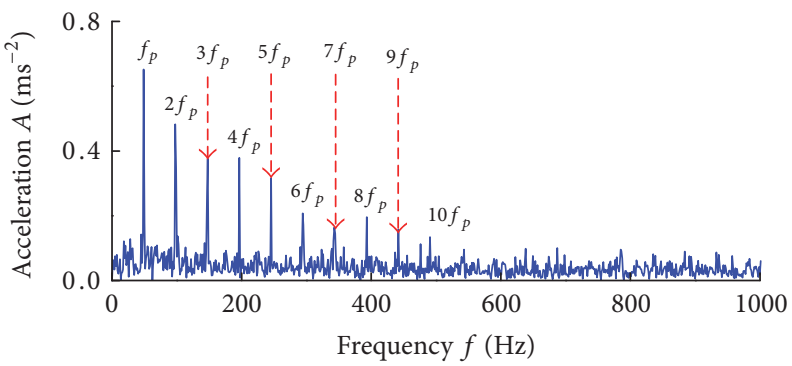

(b)

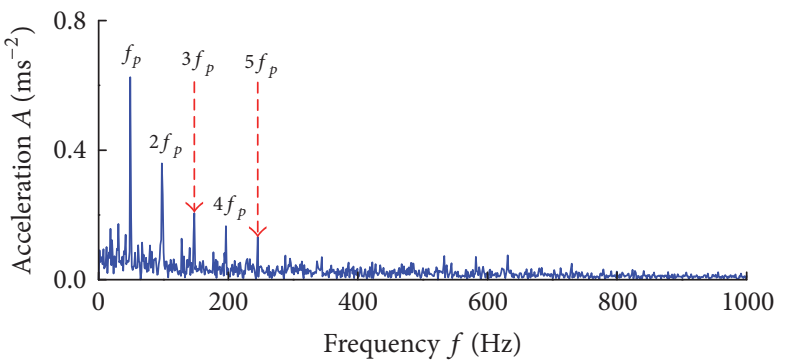

(d)

FIGURE 6: The intrinsic envelopes extracted by the manifold with Isomap and their envelope spectra: (a) on IMF-envelope space of EEMD, (b) the envelope spectra of (a), (c) on multiscale envelope space of WPT, and (d) the envelope spectra of (c). 

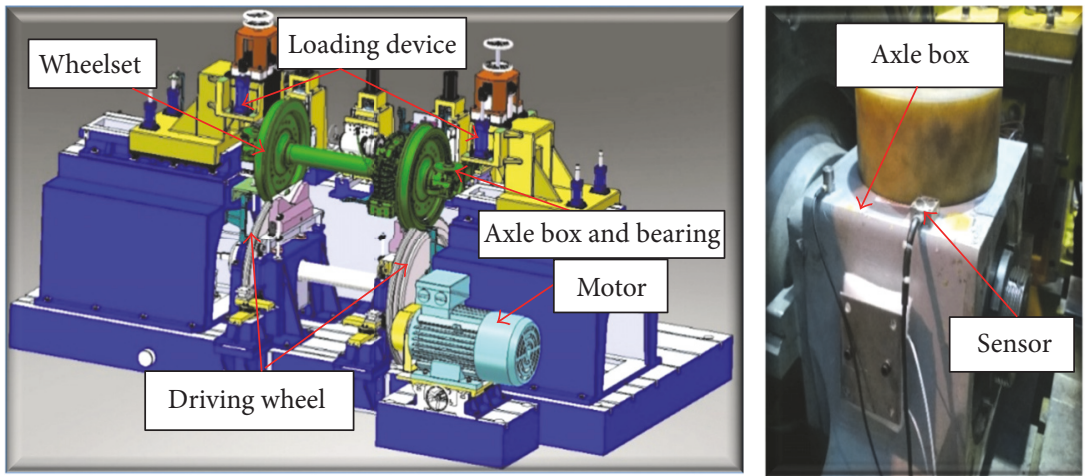

Figure 7: Photos of the test bench and measurement sensor

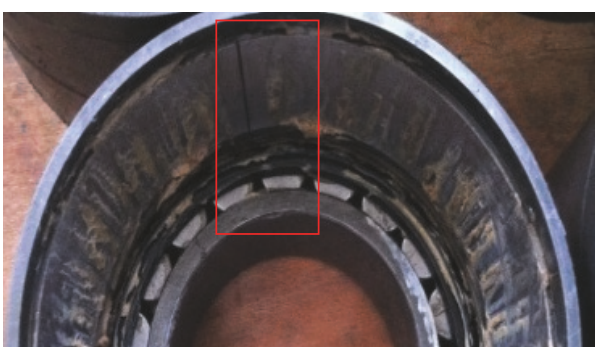

(a)

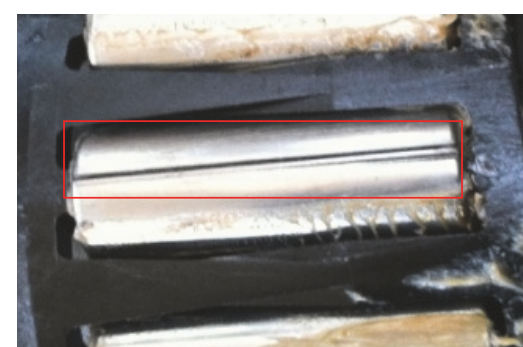

(b)

Figure 8: Photos of the artificial faults on outer race (a) and roller (b).

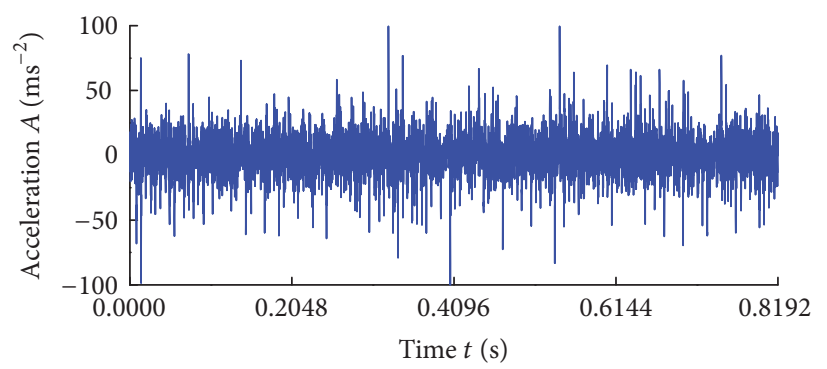

FIGURE 9: Measured vibration signals of bearing outer race fault.

fault-characteristic frequency or its harmonics. The amplitudes of the intrinsic envelope with Isomap are larger than the ones obtained by LLC. Furthermore, the amplitudes of the envelope spectra with Isomap are larger than the ones obtained with LLC. These show that the impulse-envelope manifold with Isomap is superior to the one with LLC in extracting the intrinsic envelopes with weak impulses. The fault-characteristic frequency of an outer race defect $f_{\mathrm{BPFO}}$ is expressed as

$$
f_{\mathrm{BPFO}}=\frac{N_{b}}{2}\left(1-\frac{B_{d}}{P_{d}} \cos (\phi)\right) f_{w},
$$

where $N_{b}$ is the number of rollers, $B_{d}$ is the roller diameter, $P_{d}$ is the pitch diameter, $\phi$ is the contact angle, and $f_{w}$ is the bearing rotation speed.

For comparison with the two other spaces spanned by Hilbert transform of the IMFs in EEMD and multiscale decomposition signals in WPT, the results obtained by the manifold with Isomap based on the IMFs-envelope space and multiscale envelope space are shown in Figure 11. The manifold with Isomap based on envelope of IMFs extracts the fault-characteristic frequency of the outer race fault and has 7 harmonics (the number of the extracted harmonics is the same as the impulse-envelope with Isomap and LLC). However, the manifold with Isomap based on the multiscale envelope space extracts the fault-characteristic frequency of outer race with only 2 harmonics. The amplitudes of the intrinsic envelope obtained by the manifold based on the IMF-envelope space and multiscale envelope space are less than the ones obtained by the impulse-envelope manifold with Isomap. These results illustrate that the impulseenvelope space outperforms the spaces spanned by the envelopes of IMFs and multiscale decomposition signals on extracting the impulses generated by faults on the outer race.

4.2. Results and Discussion: Fault Detection with a Fault on the Roller. The vibration signals collected from the wheelset 


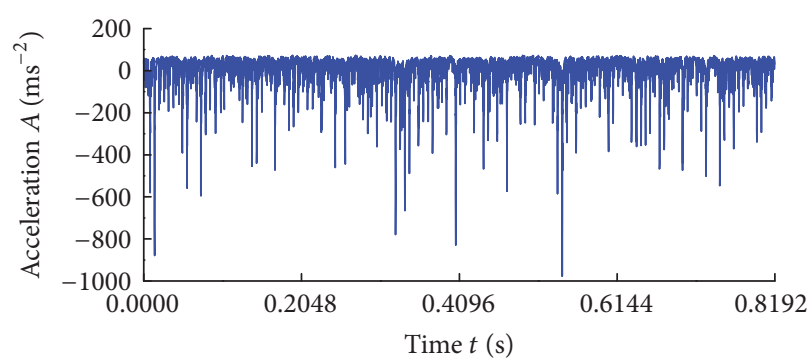

(a)

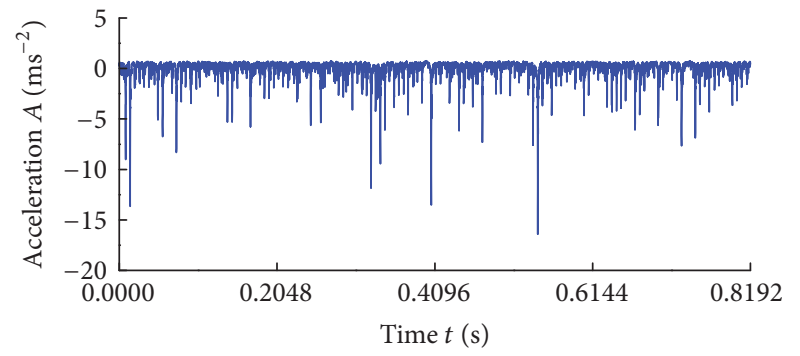

(c)

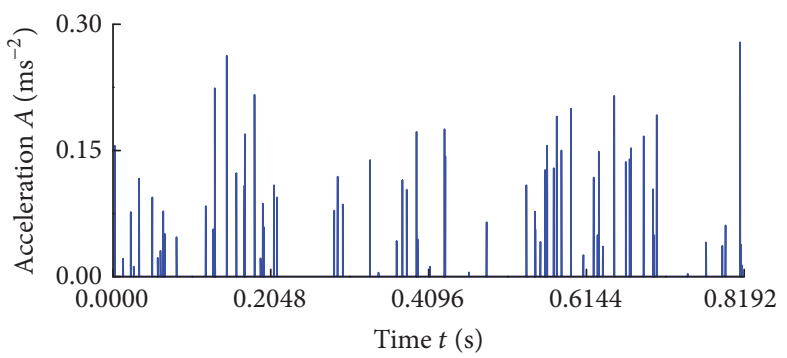

(e)

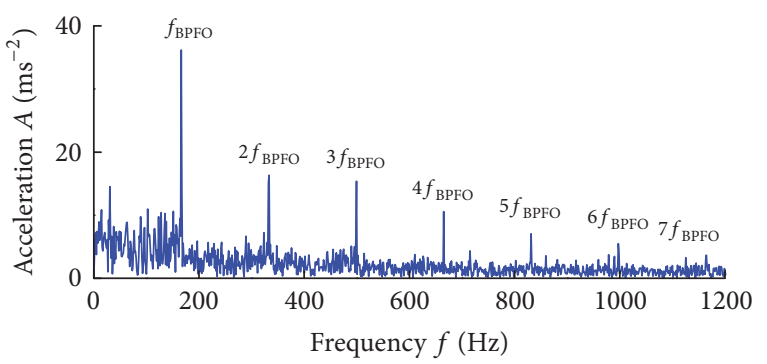

(b)

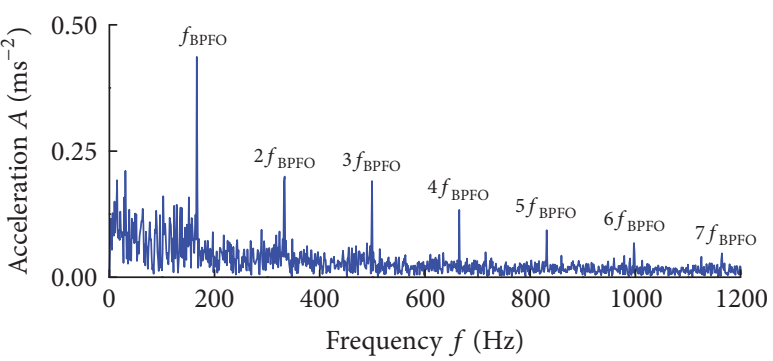

(d)

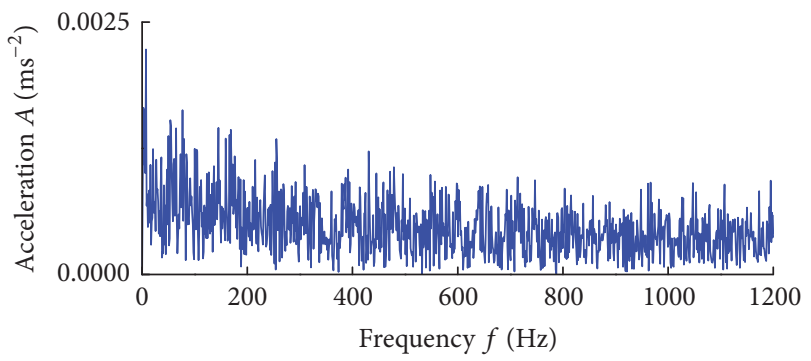

(f)

FIGURE 10: The intrinsic envelopes learned by the impulse-envelope manifold and their envelope spectra: (a) impulse-envelope with Isomap, (b) the envelope spectra of (a), (c) impulse-envelope with LLC, (d) the envelope spectra of (c), (e) impulse-envelope with LTSA, and (f) the envelope spectra of (e).

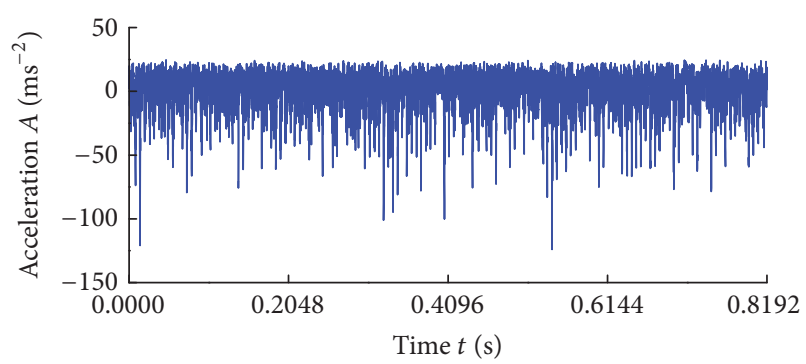

(a)

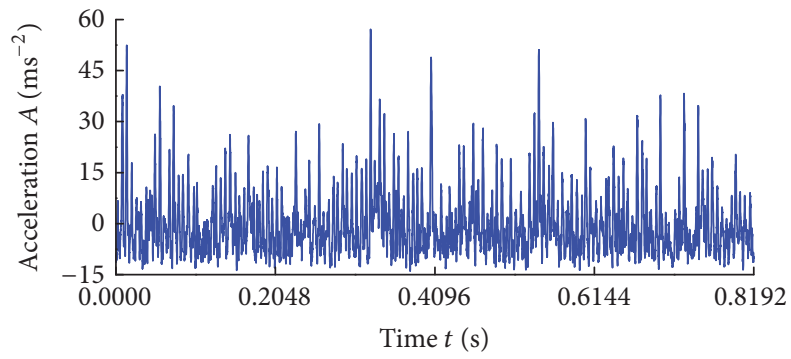

(c)

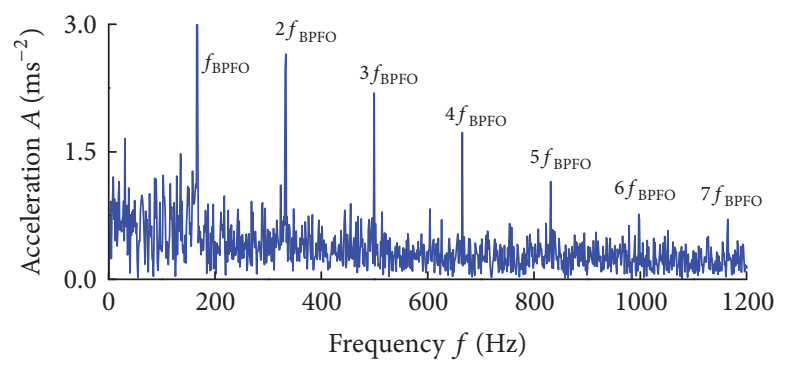

(b)

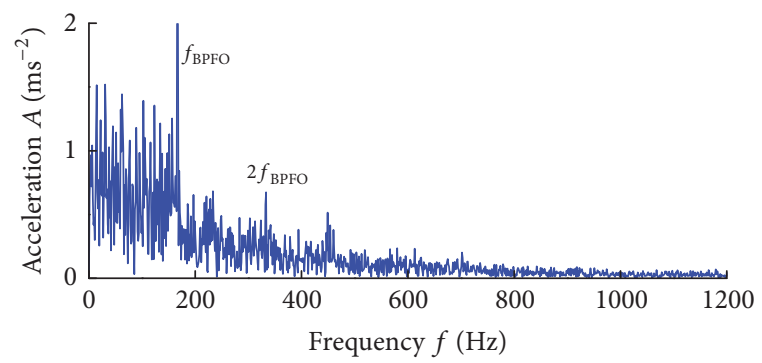

(d)

FIGURE 11: The intrinsic envelopes extracted by the manifold with Isomap and their envelope spectra: (a) IMFs-envelope space of EEMD, (b) the envelope spectra of (a), (c) multiscale envelope space of WPT, and (d) the envelope spectra of (c). 


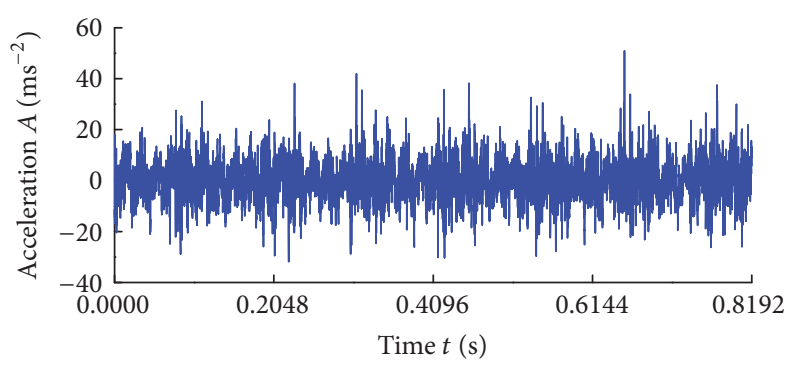

FIGURE 12: Vibration signals of a bearing roller fault.

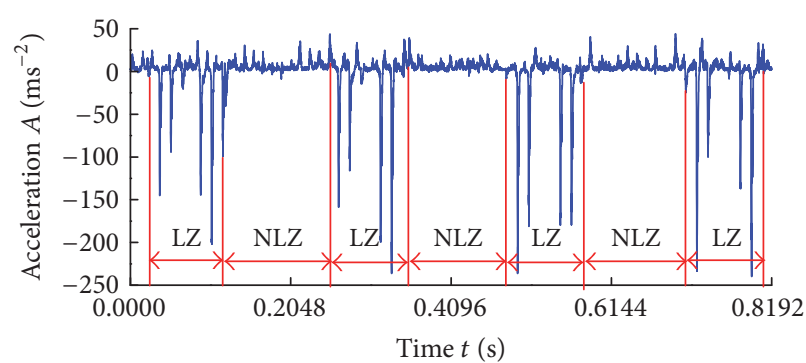

(a)

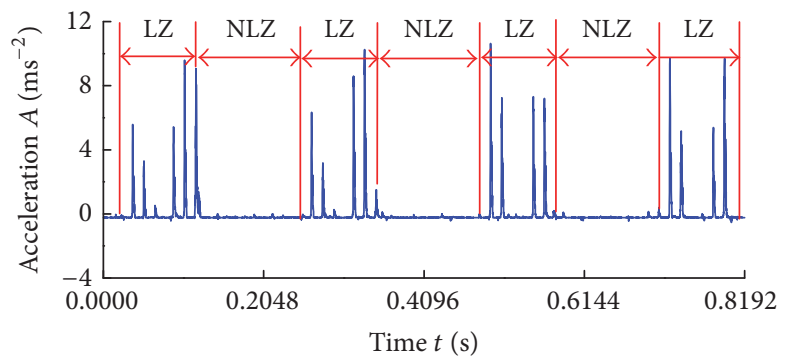

(c)

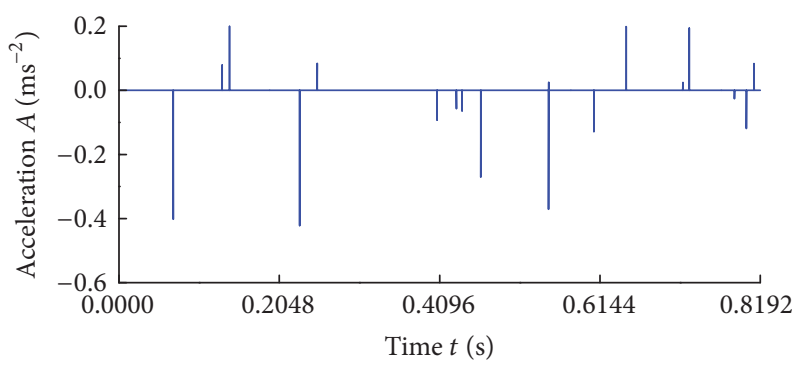

(e)

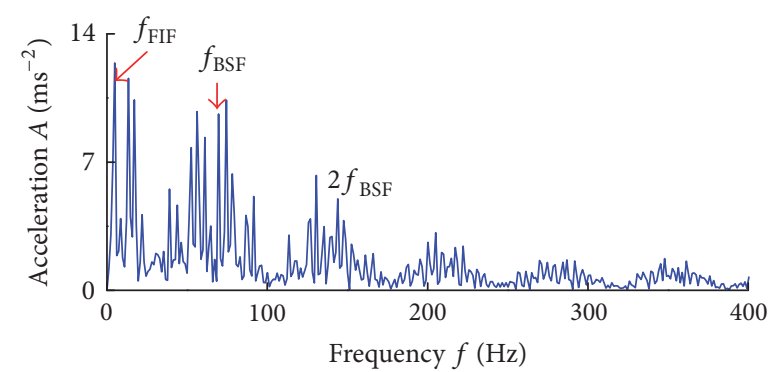

(b)

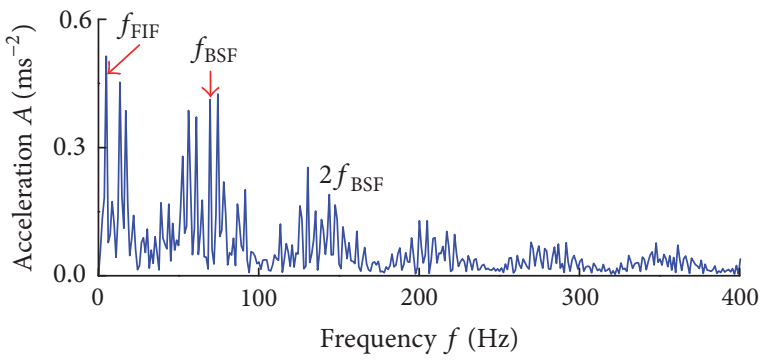

(d)

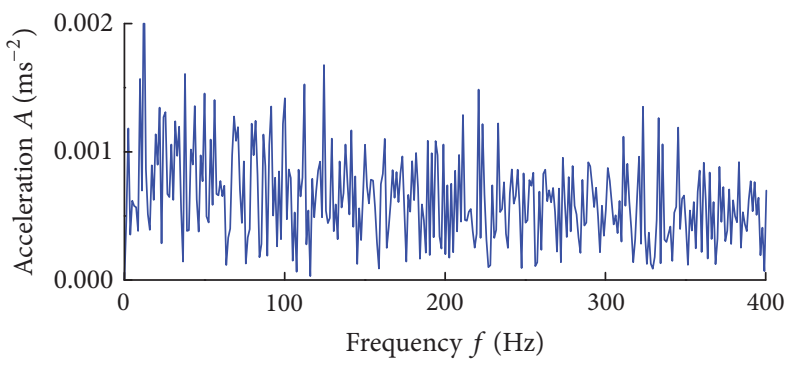

(f)

Figure 13: The intrinsic envelopes extracted by the impulse-envelope manifold and their envelope spectra: (a) the impulse-envelope with Isomap, (b) the envelope spectra of (a), (c) the impulse-envelope with LLC, (d) the envelope spectra of (c), (e) the impulse-envelope with LTSA, and (f) the envelope spectra of (e).

bearing test bench with a fault on the roller of the wheelset bearing (Figure 8(b)) are shown in Figure 12.

The intrinsic envelopes learned from the vibration signal are shown in Figure 12 and the impulse-envelope manifold is shown in Figure 13. The impulse-envelope manifold with both Isomap and LLC can well learn the low-dimensionality intrinsic envelopes from the measured vibration signals. According to the motion relationship of rollers and races shown in Figure 14, they will generate 7 impulses from
B1 to $\mathrm{H} 1$ in the load zone (LZ) of the wheelset bearing. In Figures 13(a) and 13(b), there are seven (7) impulses in the load zone of the wheelset bearing. For the wheelset bearing, the forces applied on the faulty roller are too small to excite the impulses due to the lack of contant forces in no-bearing case. In Figures 13(a) and 13(c), when the defective roller enters the LZ, there are 7 impulses. On the other hand when the defective roller leaves the LZ, there are no visible impulses. Among the seven impulses from B1 


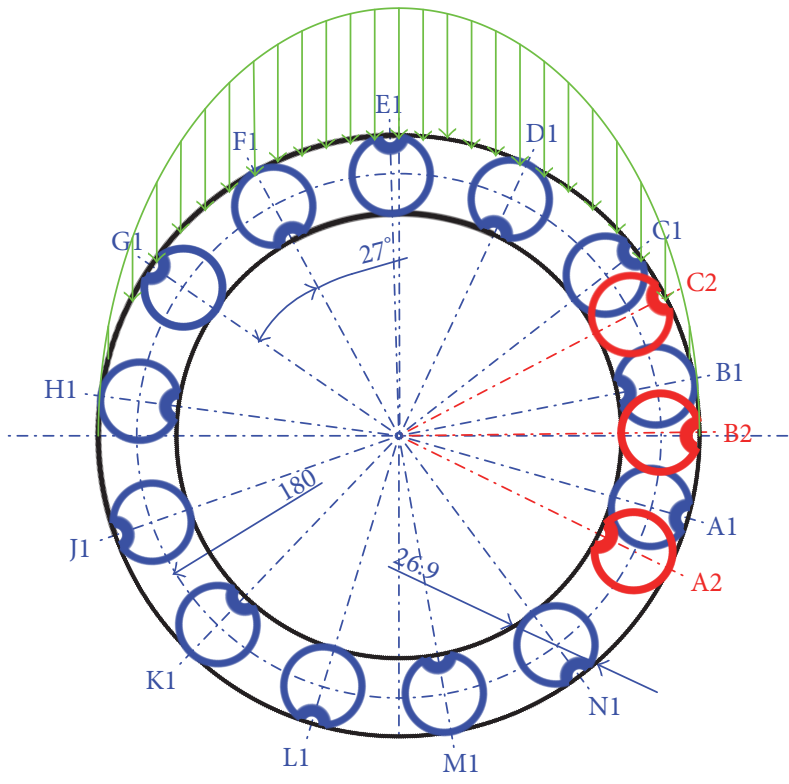

FIGURE 14: The relative motion between the rollers and races and the load zone (LZ).

to $\mathrm{H} 1$, impulse $\mathrm{B} 1$ and impulse $\mathrm{H} 1$ appear to lie at the edge of LZ of the wheelset bearing. There is a gap between the races and defective roller when the roller lies in impulse E1. The gap is caused by the symmetrical structure of the two normal rollers corresponding to impulse F1 and impulse D1. Consequently, the envelope amplitudes of impulses B1, H1, and E1 are less than those of impulses C1, D1, F1, and G1. The envelope spectra of the intrinsic envelopes obtained by impulse-envelope manifold with both Isomap and LLC can capture the fault-characteristic frequency of roller $f_{\mathrm{BPFO}}$ and its 2-order harmonics. The fault-characteristic frequency of roller $f_{\mathrm{BPFO}}$ is expressed as

$$
f_{\mathrm{BPFO}}=\frac{p_{b}}{B_{d}}\left(1-\frac{B_{d}^{2}}{P_{d}^{2}} \cos ^{2}(\phi)\right) f_{w} .
$$

On the contrary, the impulse-envelope manifold with LTSA is unable to extract the intrinsic envelopes of the vibration signals or capture the fault-characteristic frequency of roller and its harmonics. For comparison, the results obtained by the manifold with Isomap based on the IMFs-envelope space and multiscale envelope space are shown in Figure 15. However, the manifold based on the two spaces is unable to extract the intrinsic envelope of the vibration signals. The results show that the impulse-envelope manifold has excellent performance in extracting the intrinsic envelopes of the impulses induced by bearing faults.

\section{Practical Application in High-Speed Train Wheelset Bearing}

To test the capability of the proposed impulse-envelope manifold for fault detection, the technique was tested on the data obtained from an operating high-speed train. An accelerometer installed on axle box was used to gather the axle vibrations as shown in Figure 16.

5.1. Results and Discussion. Figure 17 shows the vibration signals collected from the axle box of an operating high-speed train running at a speed of $200 \mathrm{Km} / \mathrm{h}$ with a defective roller in wheelset bearing.

There are two kinds of atoms obtained by ENT. Considering atom 1 , the intrinsic envelopes learned from the vibration signals are shown in Figure 17 and after processing by the proposed impulse-envelope manifold the results are shown in Figure 18. The impulse-envelope manifold with both Isomap and LLC can successfully extract the intrinsic envelopes of the impulses induced by a roller fault. When the defective roller enters the LZ of the wheelset bearing, there are 7 impulses from impulse-envelope $B_{i}$ to impulse-envelope $H_{i}(i=$ $2,3, \ldots, 8)$. When the defective roller leaves the $\mathrm{LZ}$ of the bearing, there are no impulses due to the lack of contact force between the defective roller and races and hence no resulting envelopes appear. From the configurations of the bearing and relative motion of the rollers and the races, the intrinsic envelopes vary alternatively when the defective roller enters and leaves the LZ of the wheelset bearing as shown in Figure 14. Figure 18(e) shows that the impulse-envelope manifold with LTSA cannot extract the intrinsic envelopes of the signals. On the contrary, the intrinsic envelope spectra shown in Figures 18(b) and 18(d) can well extract the faultcharacteristic frequency $f_{\mathrm{BSF}}$ and its 3 harmonics. Figure 19 is an enlarged view from 0.12 to $0.28 \mathrm{sec}$ of Figures 18(a) and 18(b). Among the seven envelopes (B3 to H3) in Figure 19(b), the amplitude of envelope $E_{3}$ is less than other envelopes. The amplitude of the envelope $B_{3}$ is larger than envelope $C 3$ and envelope D3 and is different from the previous analysis discussed in Section 4.2. The reason is the LZ variance caused by the wheel-rail longitudinal forces to strengthen the shock effects on the defective roller at the positions of envelopes $\mathrm{B} 3$ and $\mathrm{H} 3$ during actual operation of the high-speed train. The impulse-envelope manifold with both Isomap and LLC clearly shows the novel vibration phenomenon with double envelopes of the waveform as shown in Figure 19.

Referring to atom 2, the intrinsic impulse-envelopes learned by the proposed impulse-envelope manifold are shown in Figure 20. The impulse-envelope manifolds with both Isomap and LLC can efficiently extract the envelopes of the impulses induced by the unevenness of wheelset tread. The envelope spectra in Figures 20(b) and 20(d) capture 4, 6 , and 7.5 times the rotational frequency of wheelset bearing. These show that the impulse-envelope technique provides an excellent capability of isolating the impulses generated by bearing faults and the defects of wheel tread.

For comparison, the 2-dimensionality outputs of the manifold with Isomap based on the envelope space of IMFs in EEMD are shown in Figure 21. According to prior description, two types of atoms are embedded in vibration signals shown in Figure 17 with the resulting dimensionality of the manifold set as 2 for the 2-dimensionality outputs of the manifold with Isomap based on the envelope space of multiscale decomposition signals in WPT which are shown in Figure 22. 


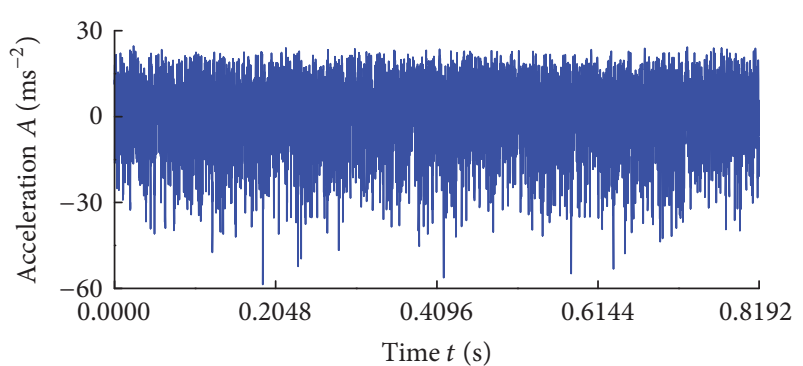

(a)

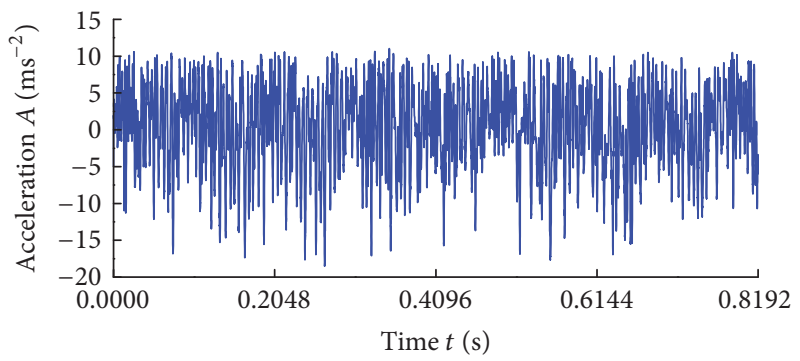

(c)

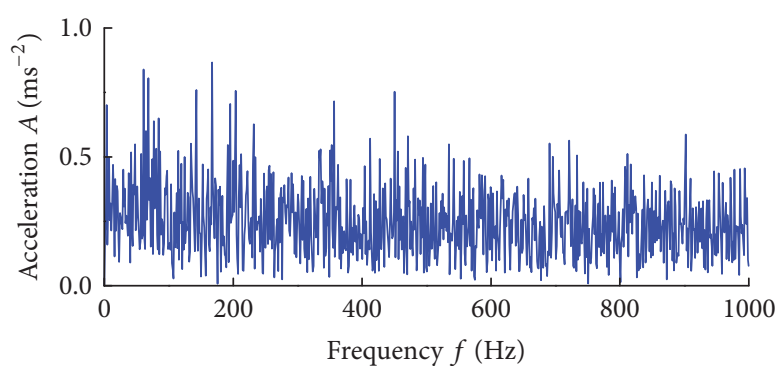

(b)

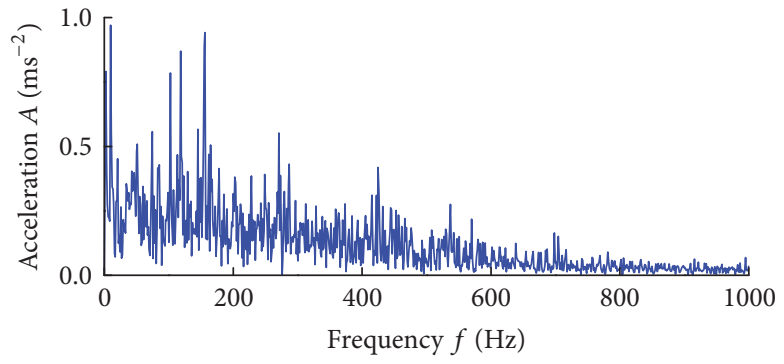

(d)

FIGURE 15: The intrinsic envelopes extracted by the manifold with Isomap and their envelope spectra: (a) IMFs-envelope space of EEMD, (b) the envelope spectra of (a), (c) multiscale envelope space of WPT, and (d) the envelope spectra of (c).

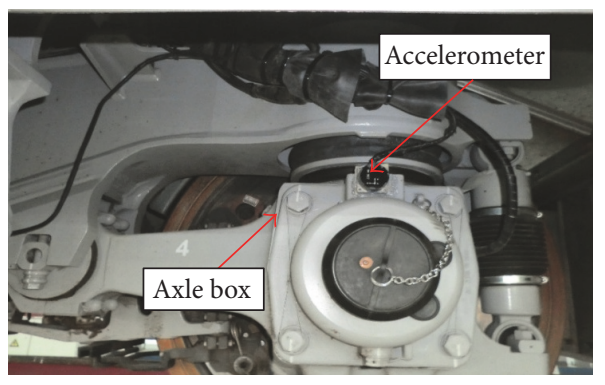

FIGURE 16: Location of accelerometer on the axle box.

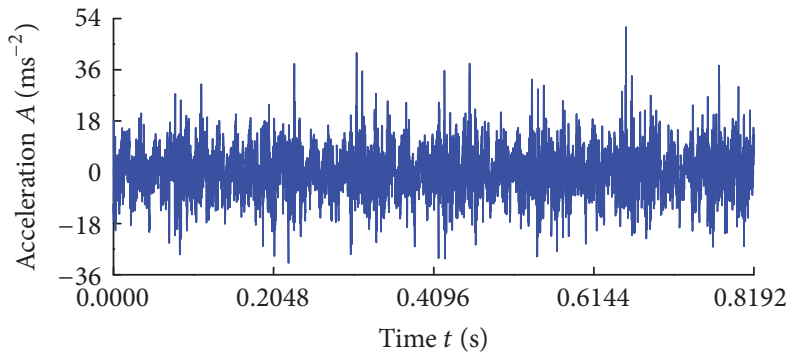

FIGURE 17: Real vibration signal obtained from the axle box.

The impulse-envelope manifold based on the two-envelope space can extract the fault-characteristic frequency $f_{\mathrm{BSF}}$ and its 2-order harmonics (less than 3-order harmonics in the impulse-envelope space) but is unable to isolate the impulses generated by wheel tread unevenness. Through comparison of Figure 21 and Figure 22, Figure 22(c) clearly exhibits the appearance of impulses. The locally zoomed figure from 0.18 to $0.26 \mathrm{sec}$ of Figure 22(c) is shown in Figure 23. The double envelopes in an envelope waveform are weakened by wavelet transform as compared to Figure 19.

\section{Conclusion}

The novelty of combining the outstanding properties of CSR and impulse-envelope manifold through Hilbert transform to extract impulses of defective roller bearings is demonstrated in this paper. Through a series of simulation 


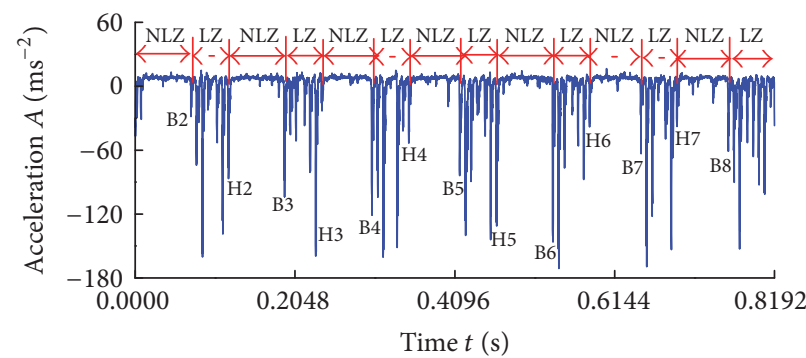

(a)

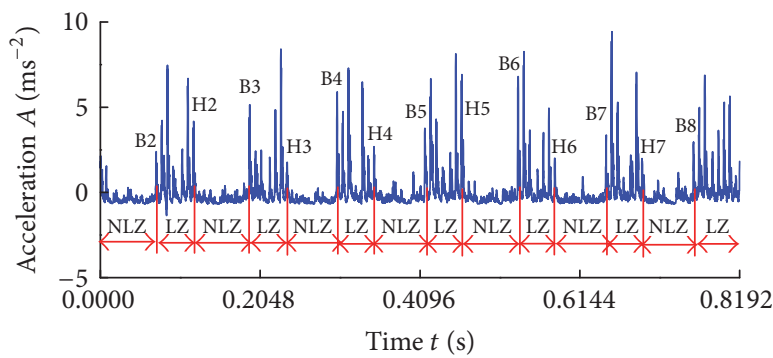

(c)

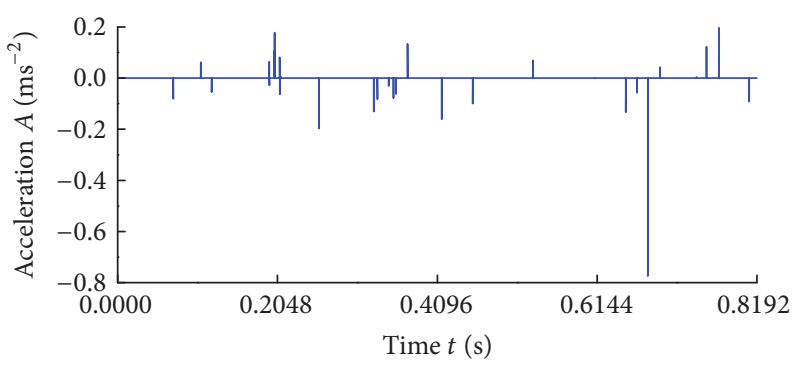

(e)

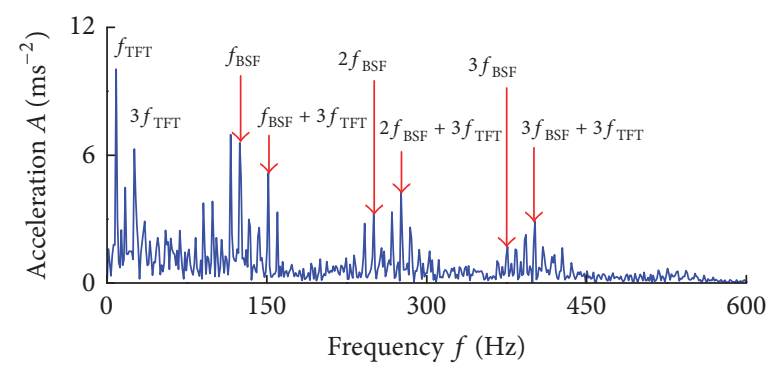

(b)

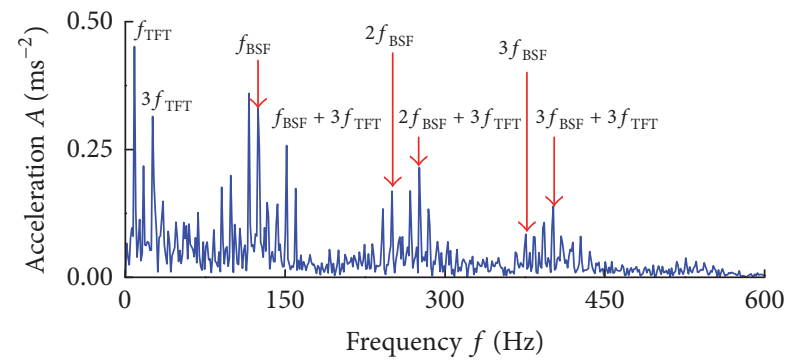

(d)

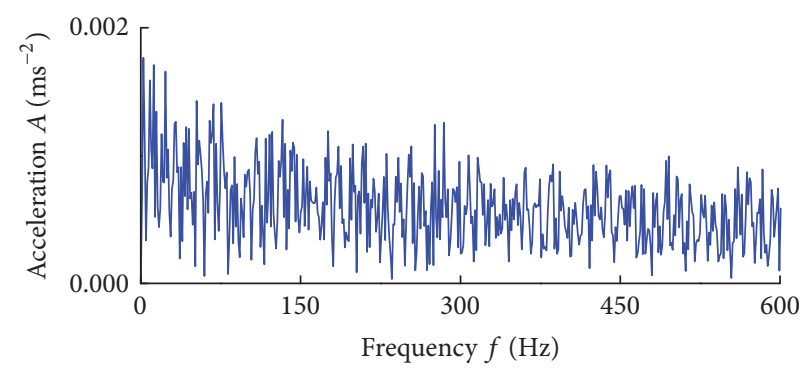

(f)

FIGURE 18: The intrinsic envelope extracted by the impulse-envelope manifold corresponding to atom 1: (a) the impulse-envelope with Isomap, (b) the Hilbert envelope spectra of (a), (c) the impulse-envelope with LLC, (d) the Hilbert envelope spectra of (c), (e) the impulse-envelope with LTSA, and (f) the Hilbert envelope spectra of (e).

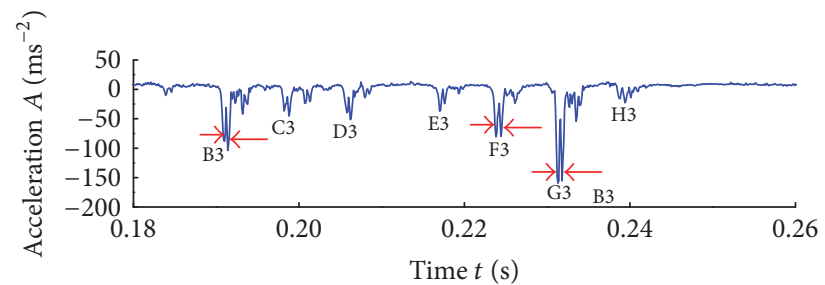

(a)

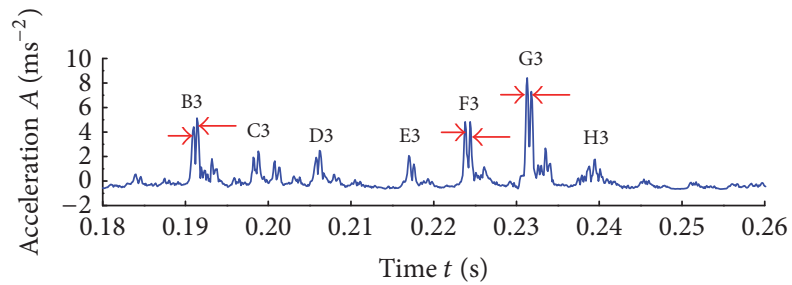

(b)

FIGURE 19: Enlarged view of intrinsic envelope from 0.18 to 0.26 sec: (a) corresponding to Figure 18(a); (b) corresponding to Figure 18(b).

studies, experimental tests, and real case data of a defective wheelset bearing of a high-speed train, the results confirm the capability of the proposed technique. The results clearly show that while both impulse-envelope manifolds with both isometric mapping (Isomap) and locally linear coordination (LLC) can successfully extract the intrinsic envelope of the impulses caused by bearing fault and unevenness wheel tread, the Isomap outperforms LCC in terms of strength and number of extracted harmonics. When comparing with EEMD and WPT, the proposed technique shows superiority in extracting the intrinsic envelope and enlarging the number of harmonics of fault-characteristic frequency.

The proposed impulse-envelope manifold also discovers a novel phenomenon for the first time of double envelope which is caused by a roller defect in an envelope waveform. This discovery is beneficial to estimate the size of the fault. However, a point to note is that the computational cost of Isomap is much higher than LLC and consequently is a topic for future research to resolve the computational cost problem. 


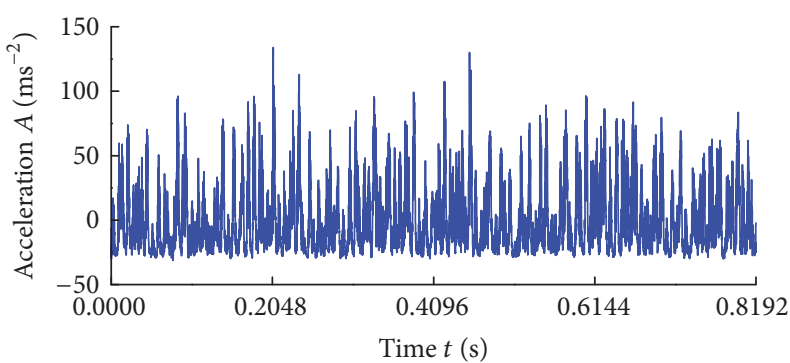

(a)

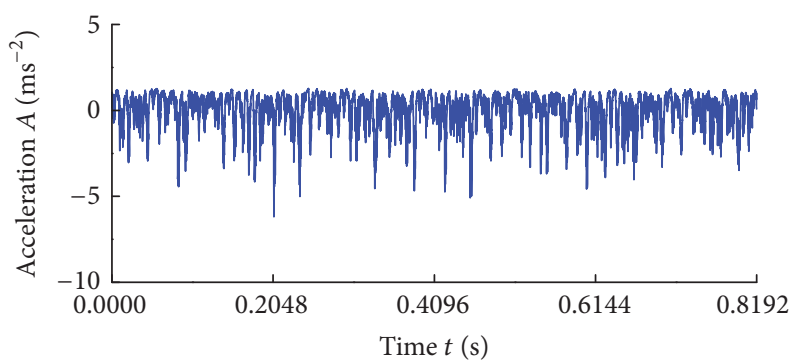

(c)

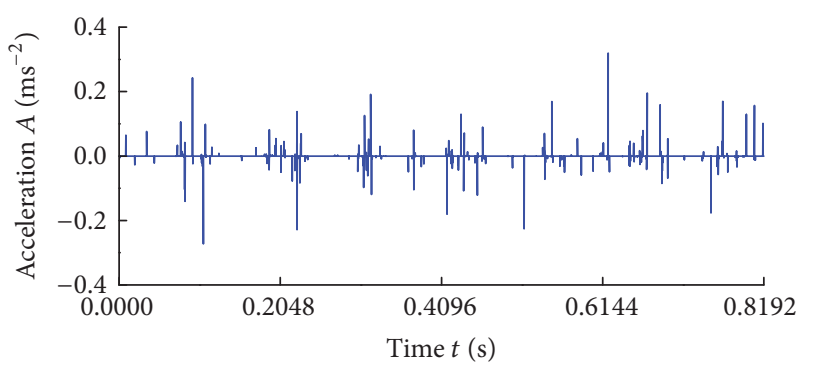

(e)

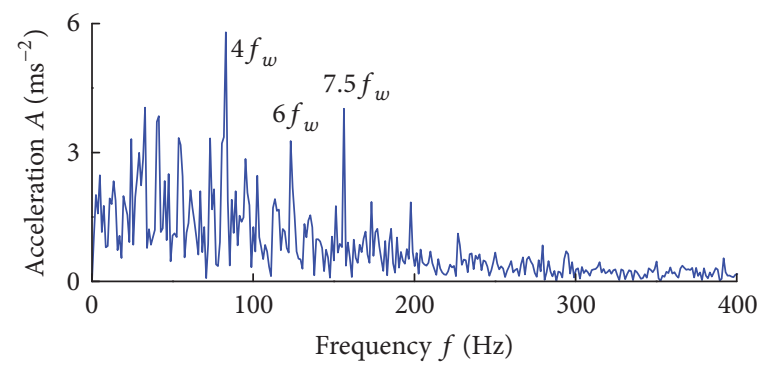

(b)

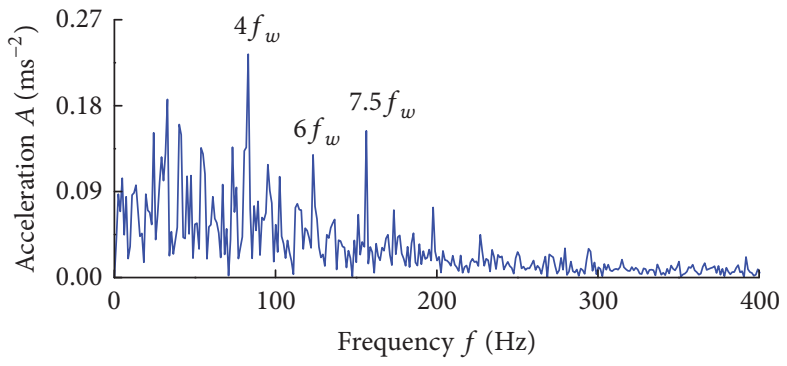

(d)

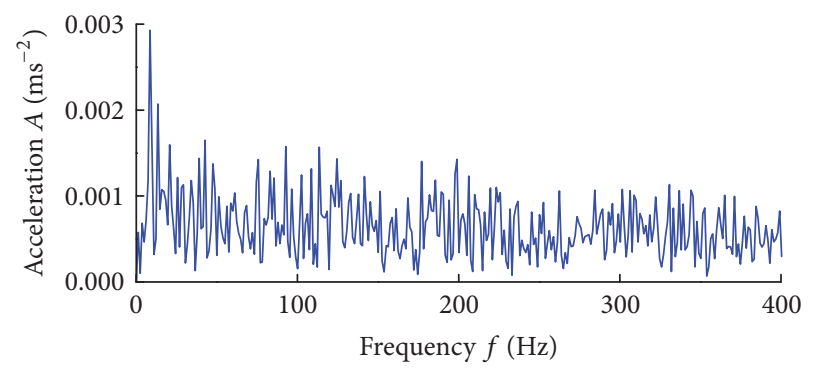

(f)

Figure 20: The intrinsic envelope of wheelset bearing vibration extracted by the impulse-envelope manifold corresponding to atom 2: (a) the impulse-envelope with Isomap, (b) the Hilbert envelope spectra of (a), (c) the impulse-envelope with LLC, (d) the Hilbert envelope spectra of (c), (e) the impulse-envelope with LTSA, and (f) the Hilbert envelope spectra of (e).

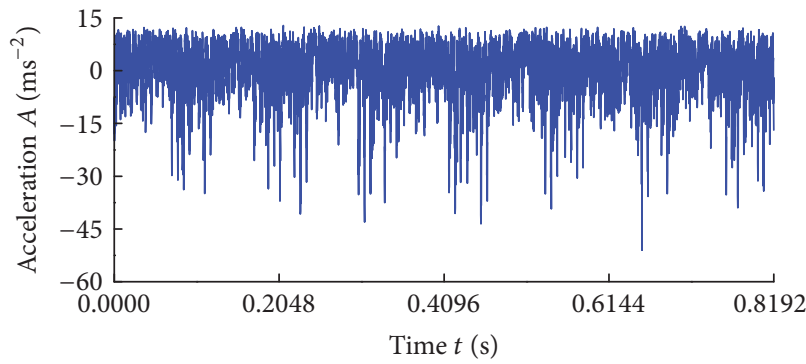

(a)

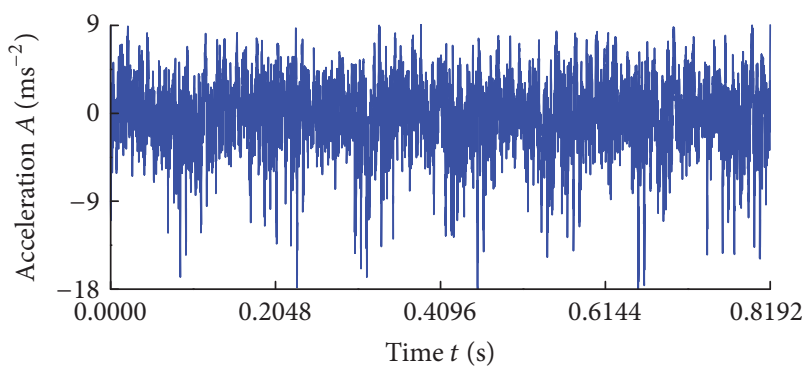

(c)

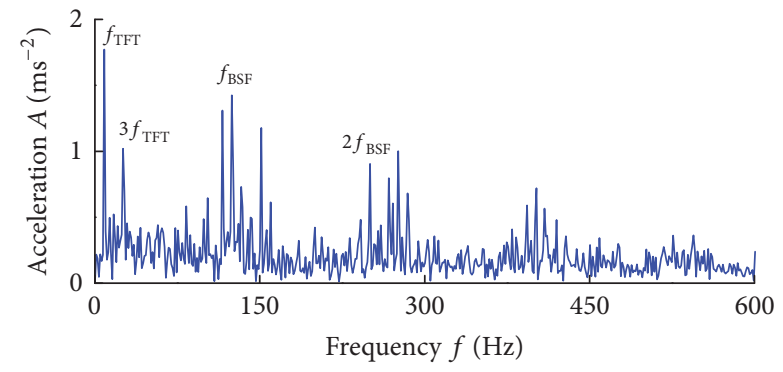

(b)

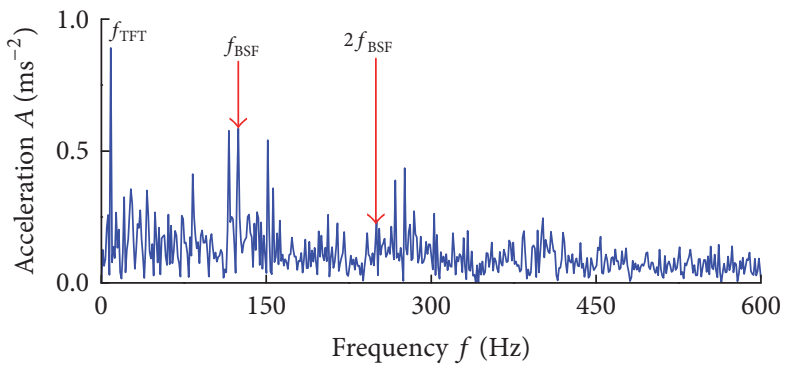

(d)

FIGURE 21: The intrinsic envelope extracted from the envelope space of IMFs: (a) corresponding to dimensionality 1, (b) the Fourier spectra of (a), (c) corresponding to dimensionality 2, and (d) the Fourier spectra of (c). 


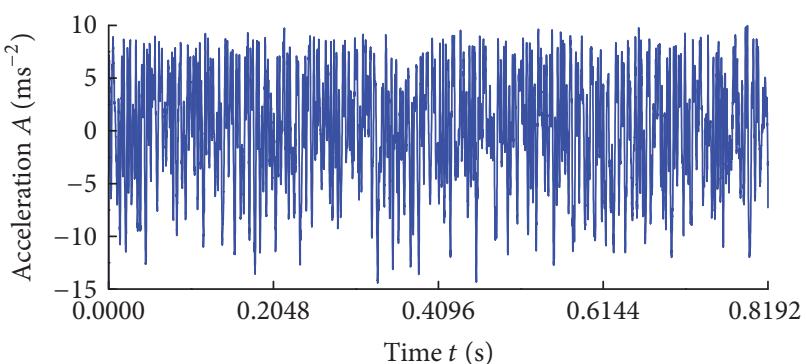

(a)

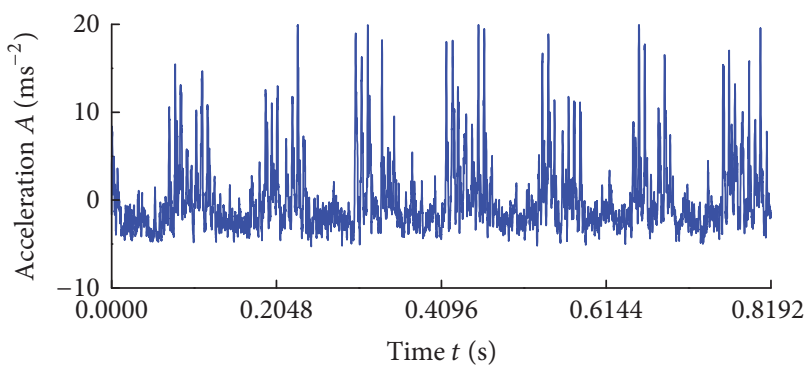

(c)

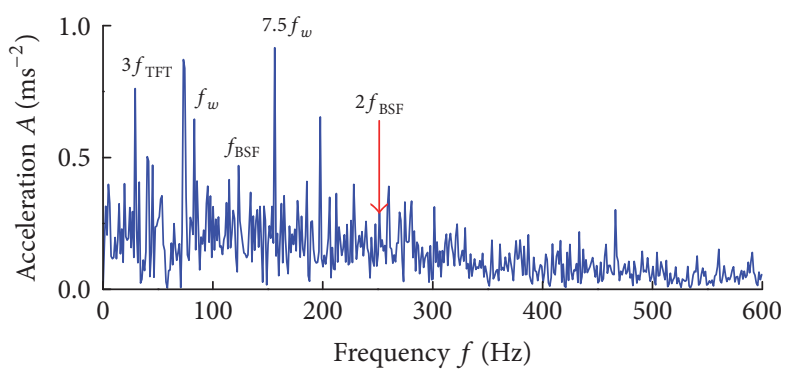

(b)

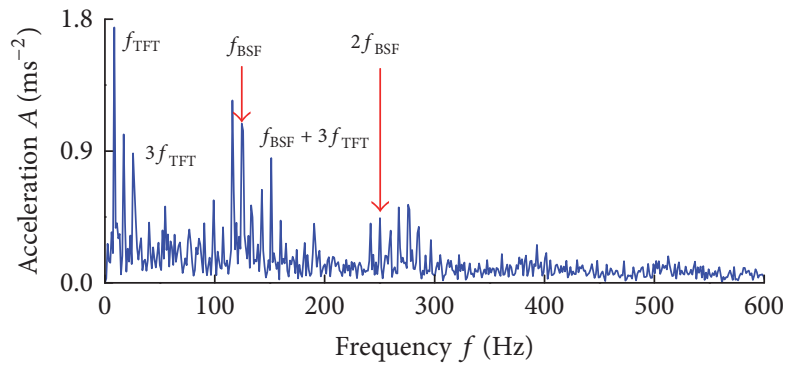

(d)

FIGURE 22: The intrinsic envelope extracted from the envelope space of multiscale decomposition signals: (a) corresponding to dimensionality of 1, (b) the Fourier spectra of (a), (c) corresponding to dimensionality of 2, and (d) the Fourier spectra.

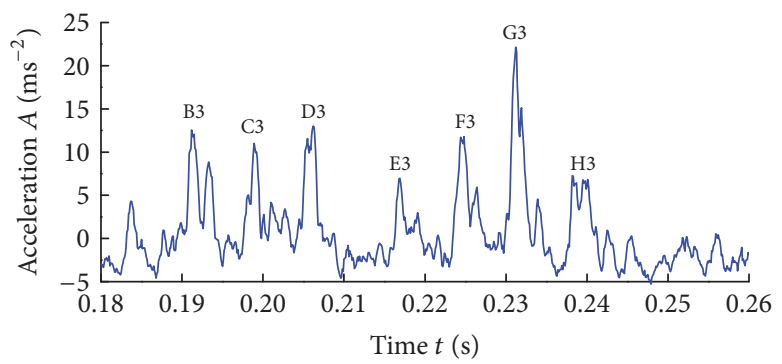

Figure 23: The locally zoomed plot of Figure 22(c).

\section{Conflicts of Interest}

The authors declare that there are no conflicts of interest regarding the publication of this paper.

\section{Acknowledgments}

This project is supported by National Natural Science Foundation of China (Grant no. 51305358), the Research Fund of State Key Laboratory of Traction Power (Grant no. 2015TPL_T17), and the Fundamental Research Funds for the Central Universities (Grant no. 2682017CX011).

\section{References}

[1] H. R. Cao, F. Fan, K. Zhou, and Z. J. He, "Wheel-bearing fault diagnosis of trains using empirical wavelet transform," Measurement, vol. 82, pp. 439-449, 2016.

[2] Z. Peng, N. J. Kessissoglou, and M. Cox, "A study of the effect of contaminant particles in lubricants using wear debris and vibration condition monitoring techniques," Wear, vol. 258, no. 11-12, pp. 1651-1662, 2005.
[3] H. Ocak, K. A. Loparo, and F. M. Discenzo, "Online tracking of bearing wear using wavelet packet decomposition and probabilistic modeling: a method for bearing prognostics," Journal of Sound and Vibration, vol. 302, no. 4-5, pp. 951-961, 2007.

[4] Y. Lei, Z. He, Y. Zi, and X. Chen, "New clustering algorithmbased fault diagnosis using compensation distance evaluation technique," Mechanical Systems and Signal Processing, vol. 22, no. 2, pp. 419-435, 2008.

[5] M. Liang and I. Soltani Bozchalooi, "An energy operator approach to joint application of amplitude and frequencydemodulations for bearing fault detection," Mechanical Systems and Signal Processing, vol. 24, no. 5, pp. 1473-1494, 2010.

[6] J. Shi, M. Liang, D.-S. Necsulescu, and Y. Guan, "Generalized stepwise demodulation transform and synchrosqueezing for time-frequency analysis and bearing fault diagnosis," Journal of Sound and Vibration, vol. 368, pp. 202-222, 2016.

[7] Q. Han and F. Chu, "Nonlinear dynamic model for skidding behavior of angular contact ball bearings," Journal of Sound and Vibration, 2015.

[8] G. He, K. Ding, and H. Lin, "Fault feature extraction of rolling element bearings using sparse representation," Journal of Sound and Vibration, vol. 366, pp. 514-527, 2016. 
[9] L. Cui, N. Wu, C. Ma, and H. Wang, "Quantitative fault analysis of roller bearings based on a novel matching pursuit method with a new step-impulse dictionary," Mechanical Systems and Signal Processing, vol. 68-69, pp. 34-43, 2016.

[10] M. Žvokelj, S. Zupan, and I. Prebil, "EEMD-based multiscale ICA method for slewing bearing fault detection and diagnosis," Journal of Sound and Vibration, vol. 370, pp. 394-423, 2016.

[11] Y. Shao and K. Nezu, "Design of mixture de-noising for detecting faulty bearing signals," Journal of Sound and Vibration, vol. 282, no. 3-5, pp. 899-917, 2005.

[12] D. Yu, J. Cheng, and Y. Yang, "Application of EMD method and Hilbert spectrum to the fault diagnosis of roller bearings," Mechanical Systems and Signal Processing, vol. 19, no. 2, pp. 259270, 2005.

[13] H. C. Wang, J. Chen, and G. M. Dong, "Feature extraction of rolling bearing's early weak fault based on EEMD and tunable Q-factor wavelet transform," Mechanical Systems and Signal Processing, vol. 48, no. 1-2, pp. 103-119, 2014.

[14] W. Su, F. Wang, H. Zhu, Z. Zhang, and Z. Guo, "Rolling element bearing faults diagnosis based on optimal Morlet wavelet filter and autocorrelation enhancement," Mechanical Systems and Signal Processing, vol. 24, no. 5, pp. 1458-1472, 2010.

[15] X. Wang, Y. Zi, and Z. He, "Multiwavelet denoising with improved neighboring coefficients for application on rolling bearing fault diagnosis," Mechanical Systems and Signal Processing, vol. 25, no. 1, pp. 285-304, 2011.

[16] L. Cui, J. Wang, and S. Lee, "Matching pursuit of an adaptive impulse dictionary for bearing fault diagnosis," Journal of Sound and Vibration, vol. 333, no. 10, pp. 2840-2862, 2014.

[17] X. F. Chen, Z. Du, J. Li, X. Li, and H. Zhang, "Compressed sensing based on dictionary learning for extracting impulse components," Signal Processing, vol. 96, pp. 94-109, 2014.

[18] W.-L. Chiang, D.-J. Chiou, C.-W. Chen, J.-P. Tang, W.-K. Hsu, and T.-Y. Liu, "Detecting the sensitivity of structural damage based on the Hilbert-Huang transform approach," Engineering Computations (Swansea, Wales), vol. 27, no. 7, pp. 799-818, 2010.

[19] R. Yan and R. X. Gao, "Hilbert-huang transform-based vibration signal analysis for machine health monitoring," IEEE Transactions on Instrumentation and Measurement, vol. 55, no. 6, pp. 2320-2329, 2006.

[20] S. A. Bagherzadeh and M. Sabzehparvar, "A local and online sifting process for the empirical mode decomposition and its application in aircraft damage detection," Mechanical Systems and Signal Processing, vol. 54, pp. 68-83, 2015.

[21] H. Jiang, C. Li, and H. Li, "An improved EEMD with multiwavelet packet for rotating machinery multi-fault diagnosis," Mechanical Systems and Signal Processing, vol. 36, no. 2, pp. 225239, 2013.

[22] F. Jiang, Z. Zhu, W. Li, G. Zhou, and G. Chen, "Fault identification of rotor-bearing system based on ensemble empirical mode decomposition and self-zero space projection analysis," Journal of Sound and Vibration, vol. 333, no. 14, pp. 3321-3331, 2014.

[23] G. Rilling, P. Flandrin, P. Goncalves, and J. M. Lilly, "Bivariate empirical mode decomposition," IEEE Signal Processing Letters, vol. 14, no. 12, pp. 936-939, 2007.

[24] J. Fleureau, A. Kachenoura, L. Albera, J.-C. Nunes, and L. Senhadji, "Multivariate empirical mode decomposition and application to multichannel filtering," Signal Processing, vol. 91, no. 12, pp. 2783-2792, 2011.

[25] J. Lin and L. Qu, "Feature extraction based on morlet wavelet and its application for mechanical fault diagnosis," Journal of Sound and Vibration, vol. 234, no. 1, pp. 135-148, 2000.
[26] Y. Qin, B. Tang, and J. Wang, "Higher-density dyadic wavelet transform and its application," Mechanical Systems and Signal Processing, vol. 24, no. 3, pp. 823-834, 2010.

[27] G. Cai, X. Chen, and Z. He, "Sparsity-enabled signal decomposition using tunable Q-factor wavelet transform for fault feature extraction of gearbox," Mechanical Systems and Signal Processing, vol. 41, no. 1-2, pp. 34-53, 2013.

[28] Y. Lei, J. Lin, Z. He, and Y. Zi, "Application of an improved kurtogram method for fault diagnosis of rolling element bearings," Mechanical Systems and Signal Processing, vol. 25, no. 5, pp. 1738-1749, 2011.

[29] Y. Lei, Z. He, and Y. Zi, "Application of the EEMD method to rotor fault diagnosis of rotating machinery," Mechanical Systems and Signal Processing, vol. 23, no. 4, pp. 1327-1338, 2009.

[30] I. W. Selesnick, R. G. Baraniuk, and N. G. Kingsbury, "The dual-tree complex wavelet transform," IEEE Signal Processing Magazine, vol. 22, no. 6, pp. 123-151, 2005.

[31] I. W. Selesnick, "Wavelet transform with tunable Q-factor," IEEE Transactions on Signal Processing, vol. 59, no. 8, pp. 3560-3575, 2011.

[32] A. M. Tillmann, "On the computational intractability of exact and approximate dictionary learning," IEEE Signal Processing Letters, vol. 22, no. 1, pp. 45-49, 2015.

[33] J. A. Tropp, "Greed is good: algorithmic results for sparse approximation," Institute of Electrical and Electronics Engineers Transactions on Information Theory, vol. 50, no. 10, pp. 22312242, 2004.

[34] M. A. T. Figueiredo, R. D. Nowak, and S. J. Wright, "Gradient projection for sparse reconstruction: application to compressed sensing and other inverse problems," IEEE Journal of Selected Topics in Signal Processing, vol. 1, no. 4, pp. 586-597, 2007.

[35] K. Engan, K. Skretting, and J. H. Husøy, "Family of iterative LS-based dictionary learning algorithms, ILS-DLA, for sparse signal representation," Digital Signal Processing, vol. 17, no. 1, pp. 32-49, 2007.

[36] H. Liu, C. Liu, and Y. Huang, "Adaptive feature extraction using sparse coding for machinery fault diagnosis," Mechanical Systems and Signal Processing, vol. 25, no. 2, pp. 558-574, 2011.

[37] H. Tang, J. Chen, and G. Dong, "Sparse representation based latent components analysis for machinery weak fault detection," Mechanical Systems and Signal Processing, vol. 46, no. 2, pp. 373388, 2014.

[38] B. Wohlberg, "Efficient algorithms for convolutional sparse representations," IEEE Transactions on Image Processing, vol. 25, no. 1, pp. 301-315, 2016.

[39] J. Ding, F. Li, J. Lin, B. Miao, and L. Liu, "Fault detection of a wheelset bearing based on appropriately sparse impulse extraction," Shock and Vibration, vol. 2017, pp. 1-17, 2017.

[40] J. B. Tenenbaum, V. de Silva, and J. C. Langford, "A global geometric framework for nonlinear dimensionality reduction," Science, vol. 290, no. 5500, pp. 2319-2323, 2000.

[41] S. Lafon and A. B. Lee, "Diffusion maps and coarse-graining: A unified framework for dimensionality reduction, graph partitioning, and data set parameterization," IEEE Transactions on Pattern Analysis and Machine Intelligence, vol. 28, no. 9, pp. 1393-1403, 2006.

[42] Z. Zhang and H. Zha, "Principal manifolds and nonlinear dimensionality reduction via tangent space alignment," SIAM Journal on Scientific Computing, vol. 26, no. 1, pp. 313-338, 2004.

[43] S. T. Roweis and L. K. Saul, "Nonlinear dimensionality reduction by locally linear embedding," Science, vol. 290, no. 5500, pp. 2323-2326, 2000. 
[44] W. Y. The and S. T. Roweis, "Automatic alignment of hidden representations," in In Advances in Neural Information Processing Systems, vol. 15, pp. 841-848, 2002.

[45] J. W. Sammon Jr., "A nonlinear mapping for data structure analysis," IEEE Transactions on Computers, vol. C-18, no. 5, pp. 401-409, 1969.

[46] J. Wang, Q. B. He, and F. R. Kong, "Automatic fault diagnosis of rotating machines by time-scale manifold ridge analysis," Mechanical Systems and Signal Processing, vol. 40, no. 1, pp. 237256, 2013.

[47] Q. He, "Vibration signal classification by wavelet packet energy flow manifold learning," Journal of Sound and Vibration, vol. 332, no. 7, pp. 1881-1894, 2013.

[48] Z. Su, B. Tang, L. Deng, and Z. Liu, "Fault diagnosis method using supervised extended local tangent space alignment for dimension reduction," Measurement, vol. 62, pp. 1-14, 2015.

[49] X. Ding and Q. He, "Time-frequency manifold sparse reconstruction: a novel method for bearing fault feature extraction," Mechanical Systems and Signal Processing, vol. 80, pp. 392-413, 2016.

[50] B. Boashash, "Estimating and interpreting the instantaneous frequency of a signal. I. Fundamentals," Proceedings of the IEEE, vol. 80, no. 4, pp. 520-538, 1992.

[51] Z. H. Wu and N. E. Huang, "Ensemble empirical mode decomposition: a noise-assisted data analysis method," Advances in Adaptive Data Analysis (AADA), vol. 1, no. 1, pp. 1-41, 2009.

[52] J.-D. Wu and C.-H. Liu, "Investigation of engine fault diagnosis using discrete wavelet transform and neural network," Expert Systems with Applications, vol. 35, no. 3, pp. 1200-1213, 2008.

[53] J. Rafiee and P. W. Tse, "Use of autocorrelation of wavelet coefficients for fault diagnosis," Mechanical Systems and Signal Processing, vol. 23, no. 5, pp. 1554-1572, 2009. 


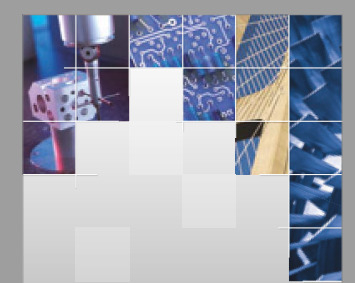

\section{Enfincering}
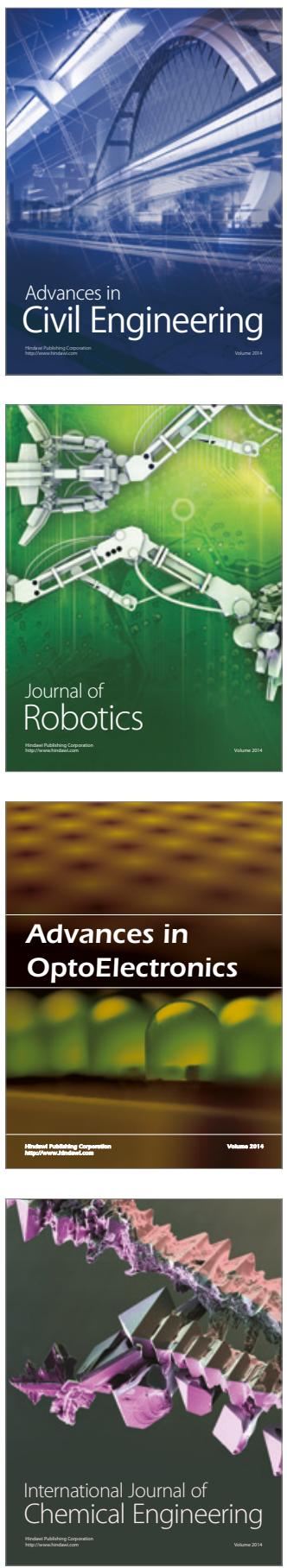

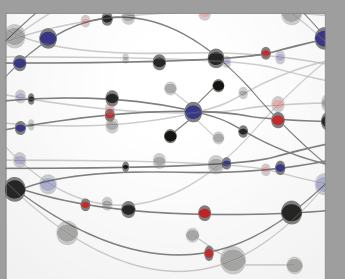

The Scientific World Journal

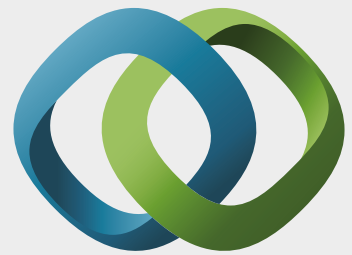

\section{Hindawi}

Submit your manuscripts at

https://www.hindawi.com
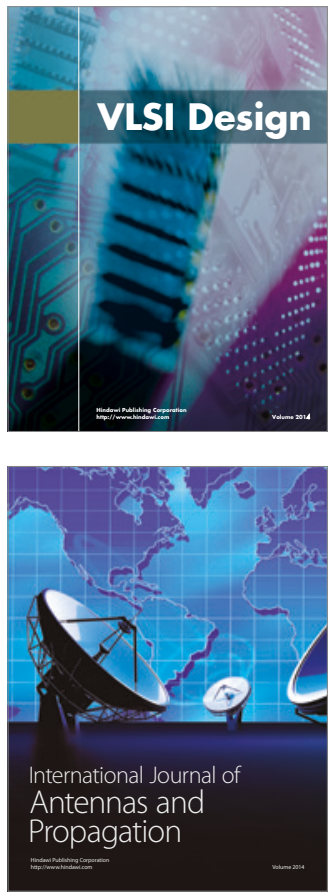

\section{Rotating}

Machinery
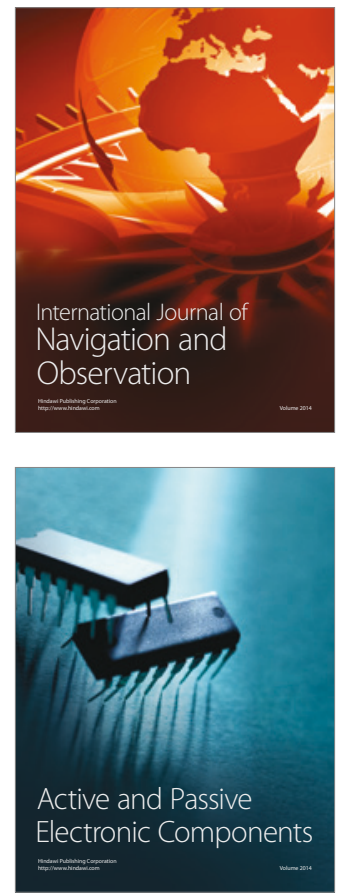
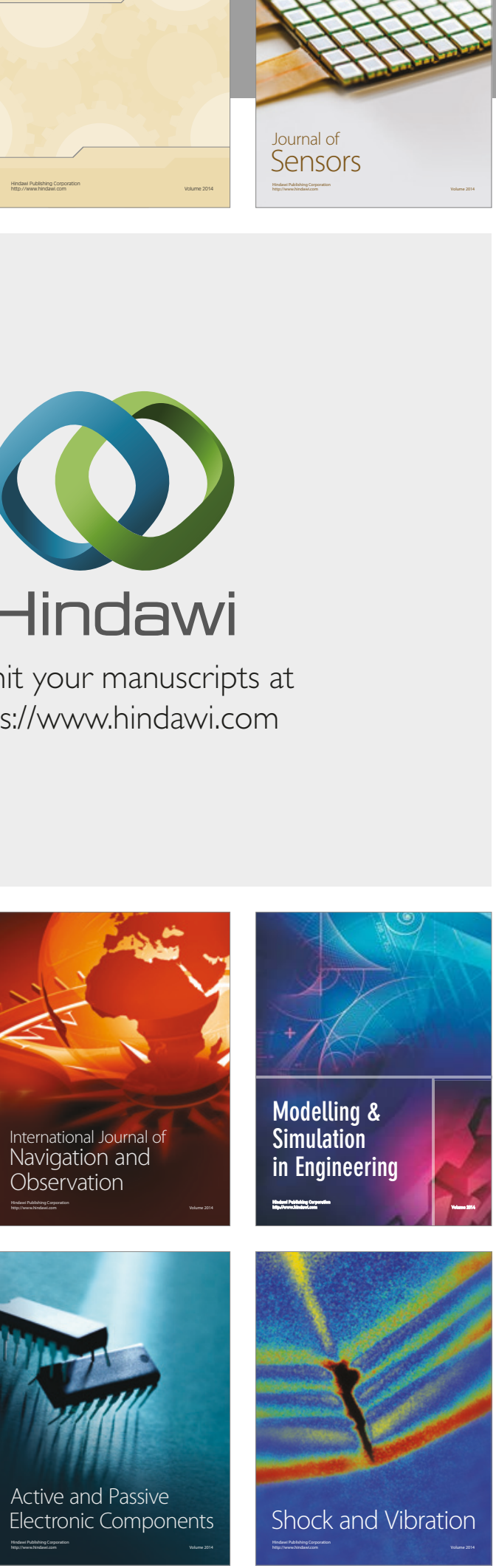
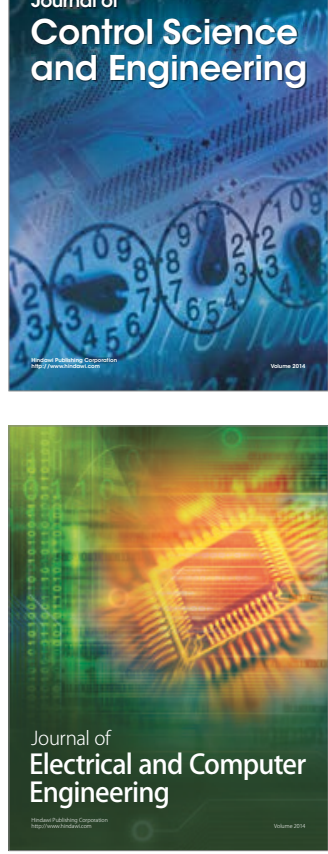

Distributed

Journal of

Control Science

and Engineering
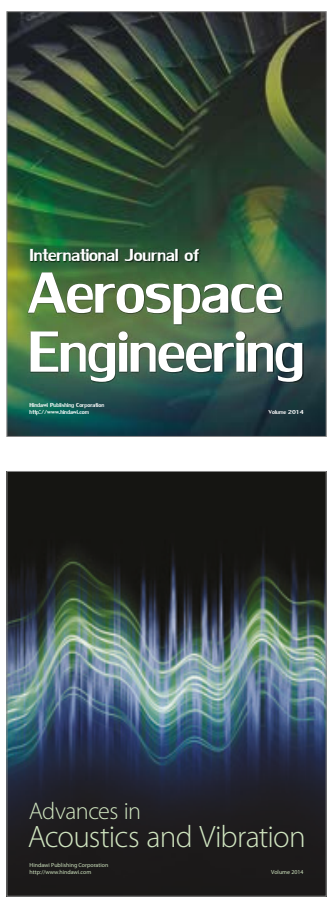

Sensor Networks 